\title{
Regularity of Local Minimizers of the Interaction Energy Via Obstacle Problems
}

\author{
J. A. Carrillo ${ }^{1}$, M. G. Delgadino ${ }^{2}$, A. Mellet $^{2}$ \\ 1 Department of Mathematics, Imperial College London, London SW7 2AZ, UK. \\ E-mail: carrillo@imperial.ac.uk \\ 2 University of Maryland, College Park, USA
}

Received: 16 June 2014 / Accepted: 23 January 2016

Published online: 17 March 2016 - () The Author(s) 2016. This article is published with open access at Springerlink.com

\begin{abstract}
The repulsion strength at the origin for repulsive/attractive potentials determines the regularity of local minimizers of the interaction energy. In this paper, we show that if this repulsion is like Newtonian or more singular than Newtonian (but still locally integrable), then the local minimizers must be locally bounded densities (and even continuous for more singular than Newtonian repulsion). We prove this (and some other regularity results) by first showing that the potential function associated to a local minimizer solves an obstacle problem and then by using classical regularity results for such problems.
\end{abstract}

\section{Introduction}

Given a pointwise defined function $W: \mathbb{R}^{N} \rightarrow(-\infty,+\infty$ ], we define the interaction energy of a probability measure $\mu \in \mathcal{P}\left(\mathbb{R}^{N}\right)$ by

$$
E[\mu]:=\frac{1}{2} \int_{\mathbb{R}^{N}} \int_{\mathbb{R}^{N}} W(x-y) d \mu(x) d \mu(y) .
$$

Here, $\mathcal{P}\left(\mathbb{R}^{N}\right)$ denotes the space of Borel probability measures, and throughout the paper, we will always assume that the interaction potential $W$ satisfies

(H1) $W$ is a non-negative lower semi-continuous function in $L_{l o c}^{1}\left(\mathbb{R}^{N}\right)$.

Under this assumption, the energy $E[\mu]$ is well defined for all $\mu \in \mathcal{P}\left(\mathbb{R}^{N}\right)$, with $E[\mu] \in$ $[0,+\infty]$. Local integrability of the potential avoids too singular potentials for which the interaction energy is infinite for many smooth densities. These very singular potentials lead to very interesting questions in crystallization [54], whose study is outside the scope of this work. Furthermore, under the assumption (H1), the potential function $\psi$ associated to a given measure $\mu$ :

$$
\psi(x):=W * \mu(x)=\int_{\mathbb{R}^{N}} W(x-y) d \mu(y)
$$


can be defined pointwise in $\mathbb{R}^{N}$, and a simple application of Fatou's lemma implies that $\psi$ is a lower semi-continuous function, see [3, Lemma 2].

The goal of this work is to investigate the regularity properties of the local minimizers of the interaction energy (1.1). For this, the keystone of this paper will be to show that the potential function $\psi(x)$ associated to a local minimizer solves an obstacle problem. This fact was suggested by the Euler-Lagrange conditions derived in [3] and will be made rigorous here in Sect. 3. Note that in order to define precisely the notion of local minimizers, we need to specify a topology on the set of probability measures. We will use here the framework developed in [3], where the authors consider local minimizers of the energy (1.1) with respect to the optimal transport distance $d_{\infty}$. We refer to Sect. 2 for the main definitions and technicalities associated to the transport distance and a brief presentation of the main results of [3].

Lots of numerical results $[1-4,18,23,32,33,56]$ show the rich structure and variety of local/global minimizers of the interaction energy by using different numerical approaches such as particle approximations, DG schemes for the gradient flow equation associated to the energy (1.1), direct resolution of the associated steady equations, radial coordinates, and so on. The interaction potentials used in most of these numerical experiments are repulsive near the origin and attractive at large distances. Typical choices are radial potentials with a unique minimum $L$ for $r>0$, decreasing (repulsive) before and increasing (attractive) after. In particular, for a system of two identical particles, the discrete energy would then be minimized when they are located at distance $L$ from each other. Particularly relevant examples are Morse potentials [5,28,38] and power-laws $[2,27,32]$.

These repulsive/attractive interaction potentials emanated from applications in selfsimilar solutions for granular media models [24,25,40], collective behavior of animals (swarming) [5,28,32,33,38,43,44], and self-assembly of nanoparticles [29,37,48,57]. Let us mention that local minimizers of the interaction energy can be seen as steady states of the aggregation equation that have been studied thoroughly for fully attractive potentials [6,20] and repulsive/attractive potentials [2-4,21,22,30-33,47], analysing qualitative properties of the evolution in different cases: finite time blow-up, stabilization towards equilibria, confinement of solutions and so on.

The beautiful result shown in [3], corroborated by the cited numerical studies, is that the support of local minimizers of the interaction energy increases as the repulsion at the origin gets stronger. In other words, concentration of particles is not allowed on small dimensional sets when the repulsion is large enough. Geometric measure theory techniques [42] were crucial to get the estimate on the dimension of the support based on the Euler-Lagrange conditions for local minimizers in transport distances. In future work, the authors intend to emulate the results of [3] by only utilizing the connection of local minimizers with the obstacle problem.

In this work, we concentrate on showing the regularity of the local minimizers for repulsive/attractive potentials behaving at the origin like

$$
W(x) \sim \frac{1}{|x|^{N-2 s}}, \quad \text { as } x \rightarrow 0 \text {, for some } s \in(0,1] \text { and } N \geq 2 \text {, }
$$

with the understanding that $W(x) \sim-\log |x|$ if $s=1$ and $N=2$, and smooth enough outside the origin. More precise statements will be given below and in Sect. 3. In other words, we will assume that the repulsion at zero is stronger or equal to Newtonian repulsion for dimension $N \geq 2$ but $W$ is still locally integrable. Let us now make a summary of the particular results known in the literature. 
As mentioned before, the case $s=1$ is of particular interest. It corresponds to Newtonian repulsion and it has received considerable attention due to its various applications. A repetitively rediscovered result in this classical case is that the global minimizer of the interaction energy for the potential

$$
W(x)=\frac{1}{|x|^{N-2}}+\frac{|x|^{2}}{2},
$$

is the characteristic function of a suitably chosen Euclidean ball. This classical result, using potential theory and capacities, was proved by Frostman [34] (but in a bounded domain instead of confinement by quadratic potentials), and it has connections with the eigenvalue distribution of random matrices $[26,46]$. This precise result can be found for instance in [41, Proposition 2.13]. In [7], the authors show that the uniform distribution in a ball is the asymptotic behavior of the corresponding gradient flow evolution. The uniqueness of the global minimizer for more singular than Newtonian repulsion, i.e.,

$$
W(x)=\frac{1}{|x|^{N-2 s}}+\frac{|x|^{2}}{2},
$$

with $0<s<1$, was obtained by Caffarelli and Vázquez via the connection to a classical obstacle problem in [16], and this strategy was also used in [50] to treat again the case $s=1$ for the evolution problem as in [7]. A generalization with external confinement potential and repulsion due to potentials more singular than Newtonian has also been recently considered in [26] because of its connection to random matrices. Let us finally mention that the case of the potential

$$
W(x)=\frac{1}{|x|^{N-2}}+\frac{|x|^{a}}{a},
$$

with $a>2$ or $2-d<a<2$ has been analysed in [32,33] showing the existence and uniqueness of compactly supported radial critical points of the interaction energy. Moreover, they show that they are monotone, bounded, and smooth functions inside their support. The monotonicity of the critical points is rather remarkable and hints to the possibility of using rearrangement techniques to prove uniqueness of absolute minimizers. Boundedness and smoothness inside the support of radial compactly supported minimizers was also proved for the so-called Quasi-Morse potentials in [23]. These Quasi-Morse potentials behave at the origin as Newtonian potentials while they exhibit similar properties to Morse potentials in terms of existence of compactly supported radial minimizers. This particular case allows for explicit computations leading to analytic expressions for these minimizers.

The main result of this paper is that for kernels satisfying (1.2), and under reasonable assumptions on $W_{a}(x)=W(x)-|x|^{2 s-N}$, local minimizers $\mu$ of the interaction energy (1.1) are absolutely continuous with respect to the Lebesgue measure, and their density function lies in $L_{l o c}^{\infty}\left(\mathbb{R}^{N}\right)$ when $s=1$ and in $C_{l o c}^{\alpha}\left(\mathbb{R}^{N}\right)$ when $s \in(0,1)$. Furthermore, we will show that for $s=1$ the density function is in $B V_{l o c}\left(\mathbb{R}^{N}\right)$ and that the support of these local minimizers is a set with locally finite perimeter. In particular, our results will apply to potentials of the form

$$
W(x)=\frac{1}{|x|^{N-2 s}}+\frac{|x|^{q}}{q}, \quad \text { for } q>0 .
$$


These results will be obtained by exploiting the connection between the Euler-Lagrange conditions for local minimizers and classical obstacle problems [13].

In fact, we will show that the potential functions of local minimizers are locally solutions of some obstacle problems. It is by using the regularity theory for the solutions of such obstacle problems $[10,11]$ that we will derive our main results on the regularity of local minimizers. Note that Newtonian repulsion $(s=1)$ will lead to the classical obstacle problem with the Laplace operator, while stronger repulsion $(s \in(0,1))$ will lead to fractional obstacle problems (with fractional power of the Laplace operator) which have been more recently studied, in particular in [14,51] (we will also use some results of $[15,16]$ where these obstacle problems arise in the study of fractional-diffusion versions of the porous medium equation).

It should be noted that potentials that are less repulsive than Newtonian (but still repulsive at the origin) also lead to some obstacle problems. However, these involve elliptic operators of higher order. A good example is the case where $V(x) \sim-|x|$ in dimension $N=3$, which leads to a biharmonic obstacle problem. The regularity theory for these higher order obstacle problems is different, and far less developed, than that of the problems considered here. It is for that reason that they will not be discussed in this paper, but will be the object of a forthcoming work.

Let us finally comment that some of our results will require some additional uniformity assumptions on the potential $W_{a}$ at infinity if the support of the local minimizer is not compact. In fact, the existence of compactly supported global miminizers for the interaction energy is a very interesting question by itself connected to statistical mechanics [49]. This property has recently been shown $[17,52]$ under natural conditions on the interaction potential $W$ related to non $H$-stability as defined in $[28,49]$.

The plan of this paper is as follows. Section 2 is devoted to describing the notion of local minimizers used in this paper and to recalling briefly the results of [3] that are relevant to our study. Section 3 gives the precise statements of the main results of this paper, both for the Newtonian and more singular than Newtonian cases. The aims of Sects. 4 and 5 are to show the main results in the Newtonian repulsion case. Section 6 is devoted to the case of more singular than Newtonian repulsions. The final section is devoted to obtaining uniqueness results in the particular case in which the attraction is a quadratic potential. A couple of technical results (mean value formulas) are presented in Appendices.

\section{Local Minimizers and Euler-Lagrange Conditions}

In this section, we give a brief summary of the main results obtained in [3]. First, in order to define our notion of local minimizers of $E[\mu]$, we have to give precise definitions of the topology that we will use in the space of probability measures. We recall that $\mathcal{P}\left(\mathbb{R}^{N}\right)$ is the set of Borel probability measures on $\mathbb{R}^{N}$ and we denote by $\mathcal{B}\left(\mathbb{R}^{N}\right)$ the family of Borel subsets of $\mathbb{R}^{N}$. The support of a measure $\mu \in \mathcal{P}\left(\mathbb{R}^{N}\right)$ is the closed set defined by

$$
\operatorname{supp}(\mu):=\left\{x \in \mathbb{R}^{N}: \mu(B(x, \epsilon))>0 \text { for all } \epsilon>0\right\} .
$$

A family of distances between probability measures have been classically introduced by means of optimal transport theory, we will review briefly some of these concepts, we refer to $[35,55]$ for further details. A probability measure $\pi$ on the product space $\mathbb{R}^{N} \times \mathbb{R}^{N}$ is said to be a transference plan between $\mu \in \mathcal{P}\left(\mathbb{R}^{N}\right)$ and $v \in \mathcal{P}\left(\mathbb{R}^{N}\right)$ if

$$
\pi\left(A \times \mathbb{R}^{N}\right)=\mu(A) \text { and } \pi\left(\mathbb{R}^{N} \times A\right)=v(A)
$$


for all $A \in \mathcal{B}\left(\mathbb{R}^{N}\right)$. If $\mu, v \in \mathcal{P}\left(\mathbb{R}^{N}\right)$, then

$$
\Pi(\mu, v):=\left\{\pi \in \mathcal{P}\left(\mathbb{R}^{N} \times \mathbb{R}^{N}\right):(2.1) \text { holds for all } A \in \mathcal{B}\left(\mathbb{R}^{N}\right)\right\}
$$

denotes the set of admissible transference plans between $\mu$ and $\nu$. Informally, if $\pi \in \Pi(\mu, v)$ then $d \pi(x, y)$ measures the amount of mass transferred from location $x$ to location $y$. Observe that $\sup _{(x, y) \in \operatorname{supp}(\pi)}|x-y|$ represents the maximum mass displacement when transporting $\mu$ onto $v$ by the transference plan $\pi$. As in [3], we will consider local minimizers of the energy functional with respect to the $\infty$-Wasserstein distance $d_{\infty}$. This distance is defined as the optimal maximal mass displacement given by

$$
d_{\infty}(\mu, v)=\inf _{\pi \in \Pi(\mu, v)} \sup _{(x, y) \in \operatorname{supp}(\pi)}|x-y|,
$$

which can take infinite values in general, but is obviously finite for compactly supported measures. This distance induces a complete metric structure restricted to the set of probability measure with finite moments of all orders, $\mathcal{P}_{\infty}\left(\mathbb{R}^{N}\right)$, as proven in [35]. By considering local minimizers with respect to this $d_{\infty}$ distance, we are only allowing small perturbations in the $d_{\infty}$ sense (corresponding to mass being moved short distances). For such perturbations, the behavior of the energy functional is close to particle-like approximations, see $[3,19]$ for more discussion related to this interpretation.

Furthermore, we note that the $d_{\infty}$-topology is the coarsest topology among all the topologies induced by transport distances. So our results will automatically hold for any local minimizers with respect to the $p$-Wasserstein distance for any $p \in[1, \infty]$. We recall that for $1 \leq p<\infty$ the distance $d_{p}$ between two measures $\mu$ and $v$ is defined by

$$
d_{p}^{p}(\mu, v)=\inf _{\pi \in \Pi(\mu, v)}\left\{\iint_{\mathbb{R}^{N} \times \mathbb{R}^{N}}|x-y|^{p} d \pi(x, y)\right\} .
$$

Note that $d_{p}(\mu, v)<\infty$ for $\mu, v \in \mathcal{P}_{p}\left(\mathbb{R}^{N}\right)$ the set of probability measures with finite moments of order $p$. Since $d_{p}(\mu, v)$ is increasing as a function of $1 \leq p<\infty$, one can show that it converges to $d_{\infty}(\mu, v)$ as $p \rightarrow \infty$. Since the distances are ordered with respect to $p$, it is obvious that the topologies are also ordered. We now can give the following definition:

Definition 2.1. We say that $\mu$ is an $\varepsilon$-local minimizer (or simply $\varepsilon$-minimizer) for the energy $E$ with respect to $d_{\infty}$, if $E[\mu]<\infty$ and

$$
E[\mu] \leq E[v]
$$

for all $v \in \mathcal{P}\left(\mathbb{R}^{N}\right)$ such that $d_{\infty}(\mu, v)<\varepsilon$.

Note that if $\mu$ is compactly supported, the previous definition is reduced to check the local minimality for compactly supported perturbations $v$. Minimizers should correspond to equilibrium configurations for the evolution equation obtained by steepest descent of the energy. However, being a functional on probability measures, the steepest descent has to be understood in the Wasserstein sense as in [45]. The transport distance to be used in that case is $d_{2}$, and the steepest descent reads as

$$
\frac{\partial \mu_{t}}{\partial t}=\operatorname{div}\left[\left(\nabla \frac{\delta E}{\delta \mu}\right) \mu_{t}\right]=\operatorname{div}\left(\mu_{t} \nabla \psi_{t}\right) \quad x \in \mathbb{R}^{N}, t>0 .
$$


For steady configurations, we expect $\nabla \psi=0$ on the support of $\mu$, due to the formal energy dissipation identity for solutions, i.e.,

$$
\frac{d}{d t} E\left[\mu_{t}\right]=-\int_{\mathbb{R}^{N}}\left|\nabla \psi_{t}\right|^{2} d \mu_{t}
$$

where $\mu_{t}$ is any solution at time $t$ of (2.2) and $\psi_{t}=W * \mu_{t}$ its associated potential. Therefore, the points on the support of a local minimizer $\mu$ of the energy $E$ should correspond to critical points of its associated potential $\psi$. This fact is made rigorous in:

Proposition 2.2 [3, Proposition 1]. Assume that $W$ satisfies (H1) and let $\mu$ be a $\varepsilon$ minimizer of the energy $E[\mu]$ in the sense of Definition 2.1. Then any point $x_{0} \in \operatorname{supp}(\mu)$ is a local minimum of $\psi=W * \mu$ in the sense that

$$
\psi\left(x_{0}\right) \leq \psi(x) \text { for a.e. } x \in B_{\varepsilon}\left(x_{0}\right) .
$$

Remark 2.3. An attentive reading of the proof of [3, Proposition 1] leads to the important observation that the $\varepsilon$ appearing in (2.3) is the same as the $\varepsilon$ appearing in Definition 2.1. In particular, it is independent of the point $x_{0}$. Moreover, only local integrability of the interaction potential is needed for that proof, i.e., there is no need of uniform local integrability of $W$ for the proof in [3, Proposition 1].

Let us also point out that the result in Proposition 2.2 for an $\varepsilon$-minimizer does not imply that its potential $\psi$ is constant in each connected component of the support of $\mu$. Additional information is needed in terms either of the regularity of the potential $\psi$ (continuity of $\psi$, see next section), or in terms of the interaction potential $W$ itself (see [3, Proposition 2]). Another possibility is to change the topology. In fact, as proven in [3, Theorem 4], if $\mu$ is a $d_{2}$-local minimizer of the energy, then the potential $\psi$ satisfy the Euler-Lagrange conditions given by

(i) $\psi(x)=(W * \mu)(x)=2 E[\mu] \mu$-a.e.

(ii) $\psi(x)=(W * \mu)(x) \leq 2 E[\mu]$ for all $x \in \operatorname{supp}(\mu)$.

(iii) $\psi(x)=(W * \mu)(x) \geq 2 E[\mu]$ for a.e. $x \in \mathbb{R}^{N}$.

These conditions simplify to

$$
\begin{array}{ll}
\psi(x)=(W * \mu)(x)=2 E[\mu] \text { for a.e. } & x \in \operatorname{supp}(\mu), \\
\psi(x)=(W * \mu)(x) \geq 2 E[\mu] \text { for a.e. } & x \in \mathbb{R}^{N} \backslash \operatorname{supp}(\mu),
\end{array}
$$

if $\mu$ is absolutely continuous with respect to the Lebesgue measure. This problem is already quite close to classical obstacle problems encountered in semiconductors [13]. In fact, we leave to the interested reader to check that the particular case

$$
W(x)=\frac{1}{|x|^{N-2}}+\frac{|x|^{2}}{2}
$$

with $N \geq 3$, coincides precisely with the problem solved in [13], see also [12].

Now, let us connect the qualitative results in [3] to the present work. The main result in [3] shows that the support of $\varepsilon$-minimizers for potentials that are $\beta$-repulsive at the origin gets larger and larger as the repulsion gets stronger and stronger at the origin. To be more precise if the potential satisfies

$$
-\Delta W(x) \geq \frac{C}{|x|^{\beta}} \quad \text { for } 0<\beta<N
$$


at the origin, see [3, Definition 2] for a more precise statement, then any $\varepsilon$-minimizer of $E$ has the property that the Hausdorff dimension of its support is greater than or equal to $\beta$. We observe that the notion of $\beta$-repulsivity with $2 \leq \beta<N$ at the origin in [3, Definition 2] implies, in particular, that $W(0)=+\infty$ for singular at zero potentials, avoiding trivial minimizers, see [3, Sect. 3.3]. In this work, we will show that when the potential at the origin is even more repulsive, i.e., it satisfies (1.2) in a sense to be made precise in the next section, then the $\varepsilon$-minimizers must be very regular and smooth. This regularity will emanate from the obstacle-like problem to which the Euler-Lagrange conditions written above are equivalent to. Observe that when the potential satisfies (1.2), we are saying in some sense that the potential is $\beta$-repulsive with $N \leq \beta<N+2$.

\section{Main Results and Strategy}

In this section, we will first discuss in details the Newtonian case $s=1$ and then turn to the case $s \in(0,1)$. Let us denote by $\omega_{N}$ the area of the $N$-dimensional unit sphere. We denote by

$$
V(x)= \begin{cases}\frac{1}{N(N-2) \omega_{N}} \frac{1}{|x|^{N-2}} & \text { if } N \geq 3 \\ \frac{1}{2 \pi} \log \frac{1}{|x|} & \text { if } N=2\end{cases}
$$

the fundamental solution of Laplace equation, which satisfies

$$
-\Delta V=\delta_{0}
$$

Without loss of generality (we can always multiply $W$ by a constant without changing the minimizers), we will assume that

(H2) The function $W_{a}(x):=W(x)-V(x)$ satisfies:

$$
\Delta W_{a} \in L_{l o c}^{p}\left(\mathbb{R}^{N}\right) \quad \text { for some } p \in\left(\frac{N}{2}, \infty\right]
$$

and

$$
\Delta W_{a}(x) \geq-C_{*} \quad \text { a.e. } x \in \mathbb{R}^{N} .
$$

We use the notation $W_{a}$, because it is convenient to think that $W=V+W_{a}$ where $V$ describe the repulsive interactions, while $W_{a}$ describes the attractive interactions. But of course $W_{a}$ could include both repulsive and attractive effects. However, assumption (H2) ensures that the dominant behavior near 0 is Newtonian repulsion. Without loss of generality, we will assume that $W(0)=+\infty$ to avoid trivial local minimizers given by Dirac Delta at a point. Note that Sobolev embeddings theorems imply that $W_{a}$ is a Hölder continuous function in $\mathbb{R}^{N}$.

Remark 3.1. Hypothesis (H2) is in particular satisfied by the power potentials $W_{a}(x)=$ $|x|^{q} / q$ with $q>0$. Indeed, we have $\Delta W_{a}=(q+N-2)|x|^{q-2}$ which is bounded below (in fact, we have $\Delta W_{a} \geq 0$ provided $q>2-N$ ). Furthermore, we see that $\Delta W_{a} \in L_{\text {loc }}^{\infty}\left(\mathbb{R}^{N}\right)$ if $q \geq 2$, and $\Delta W_{a} \in L_{\text {loc }}^{p}\left(\mathbb{R}^{N}\right)$ for all $p<\frac{N}{2-q}$, if $q<2$. In particular, (3.2) holds for all $q>0$. 
When $\mu$ is not compactly supported, the fact that $\Delta W_{a}$ is only locally in $L^{p}\left(\mathbb{R}^{N}\right)$ will be problematic, and we will need to assume that $\Delta W_{a}$ is locally uniformly in $L^{p}\left(\mathbb{R}^{N}\right)$.

More precisely, we always assume that one of the following holds: either

(H3a) The support of $\mu, \operatorname{supp}(\mu)$, is compact in $\mathbb{R}^{N}$

or

(H3b) $\Delta W_{a}$ is locally uniformly in $L^{p}\left(\mathbb{R}^{N}\right)$ for some $p \in\left(\frac{N}{2}, \infty\right]$, that is there exists a constant $M$ such that

$$
\left\|\Delta W_{a}\right\|_{L^{p}\left(B_{1}(x)\right)} \leq M \quad \text { for all } x \in \mathbb{R}^{N} .
$$

Furthermore, in dimension $N=2$, we assume that $\mu$ is such that

$$
\int_{\mathbb{R}^{N}} \log (1+|x|) d \mu<\infty .
$$

We note that (H3b) holds typically for potential that do not grow too much at $\infty$, while it is expected that for potentials that grow fast enough at $\infty$, local minimizers of the energy have compact support, i.e. (H3a) should hold (this last fact remains to be proved though). So conditions (H3a) and (H3b) should be seen as complementary. We recall also that the existence of compactly supported global minimizers of the interaction energy $E$ has recently been proved in $[17,52]$ under natural conditions on the interaction potential related to non $H$-stability as defined in $[28,49]$. Thus, relevant minimizers, in applications such as swarming [2,3,5,23,28,32,33], are typically compactly supported.

When $\operatorname{supp}(\mu)$ is compact, say $\operatorname{supp}(\mu) \subset B_{R}(0)$, we can cut-off the kernel $W$ in a smooth way outside the ball $B_{4 R}(0)$. The density $\mu$ will still be an $\epsilon$-minimizer of the energy $E$ and its potential $\psi$ will be unchanged in the ball $B_{2 R}(0)$. So whenever assuming (H3a), it is possible to replace (3.2) with $\Delta W_{a} \in L^{p}\left(\mathbb{R}^{N}\right)$. In conclusion, whether (H3a) or (H3b) is satisfied, we can always assume that (3.4) holds.

We now have all the assumptions that we will need on the interaction potential in the case of Newtonian repulsion. We will later see how those conditions must be changed for more repulsive potential (see Sect. 3.3). But first, let us give the main results in this Newtonian case.

3.1. An obstacle problem. As mentioned earlier, the keystone of this paper is the observation that the potential function $\psi$ solves (locally) an obstacle problem. In order to make this fact rigorous, we will first need to prove that $\psi$ is a continuous function. Our first result is thus the following proposition:

Proposition 3.2 (Continuity of the potential). Let $\mu$ be an $\varepsilon$-minimizer of $E$ in the sense of Definition 2.1, and assume that $(\mathrm{H} 1),(\mathrm{H} 2)$, and either $(\mathrm{H} 3 \mathrm{a})$ or $(\mathrm{H} 3 \mathrm{~b})$ hold. Then the potential $\psi(x):=W * \mu(x)$ associated to $\mu$ is a continuous function in $\mathbb{R}^{N}$.

This proposition will be proved in Sect. 4. As a consequence, we can now show that $\psi$ is locally the solution of an obstacle problem in the neighborhood of any point in the support of $\mu$. Indeed, under the conditions (H1)-(H2)-(H3x), using both Propositions 2.2 and 3.2, we see that for any point $x_{0} \in \operatorname{supp}(\mu)$

$$
\psi(x) \geq \psi\left(x_{0}\right) \text { for all } x \in B_{\varepsilon}\left(x_{0}\right)
$$


holds (see Corollary 4.3). Furthermore, since $\varepsilon$ does not depend on $x_{0}$ (see Remark 2.3), this implies

$$
\psi(x)=\psi\left(x_{0}\right) \quad \text { in } B_{\varepsilon}\left(x_{0}\right) \cap \operatorname{supp}(\mu) .
$$

Next, we observe that (3.1) implies

$$
-\Delta \psi=\mu-\Delta W_{a} * \mu \quad \text { in } \mathcal{D}^{\prime}\left(\mathbb{R}^{N}\right)
$$

where (using (H3) and Minkowski's integral inequality), $\Delta W_{a} * \mu \in L_{l o c}^{p}\left(\mathbb{R}^{N}\right)$. In particular, since $\mu$ is a non-negative measure, we deduce

$$
-\Delta \psi \geq-\Delta W_{a} * \mu \quad \text { in } B_{\varepsilon}\left(x_{0}\right) .
$$

Furthermore, if $x \in B_{\varepsilon}\left(x_{0}\right)$ is such that $\psi(x)>\psi\left(x_{0}\right)$, (3.6) implies that $x \notin \operatorname{supp}(\mu)$, and so (by definition of $\operatorname{supp}(\mu)), \mu\left(B_{r}(x)\right)=0$ for some small $r>0$. We deduce

$$
-\Delta \psi=-\Delta W_{a} * \mu \quad \text { in } \mathcal{D}^{\prime}\left(\mathbb{R}^{N}\right) \text { in } B_{\varepsilon}\left(x_{0}\right) \cap\left\{\psi>\psi\left(x_{0}\right)\right\} .
$$

We thus have the following proposition:

Proposition 3.3. For all $x_{0} \in \operatorname{supp}(\mu)$, the potential function $\psi$ is equal, in $B_{\varepsilon}\left(x_{0}\right)$, to the unique solution of the obstacle problem

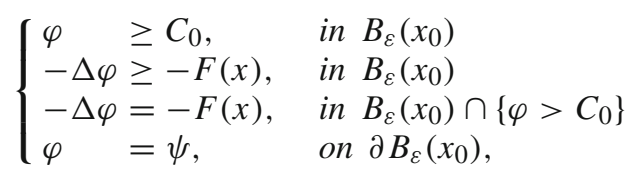

where $C_{0}=\psi\left(x_{0}\right)$ and $F(x)=\Delta W_{a} * \mu \in L_{l o c}^{p}\left(\mathbb{R}^{N}\right)$. Furthermore, the measure $\mu$ is given by

$$
\mu=-\Delta \psi+F
$$

We just showed that $\psi$ solves (3.7). For the uniqueness, we refer to [39]. Since $F$ depends on $\mu$ itself, it seems difficult to exploit (3.7) to identify local minimizers or to prove global properties such as uniqueness or radial symmetry. However, because $F$ is more regular than $\mu$, we will be able to use (3.7) to study the regularity of these local minimizers.

We also insist here on the fact that in general the constant $C_{0}$ might depend on the choice of $x_{0} \in \operatorname{supp}(\mu)$. For global minimizers, as well as for local $d_{2}$ minimizers, this constant is actually independent of $x_{0}$ as discussed in the previous section, see [3, Theorem 4].

The Eq. (3.8) suggests that there is a relation between the support of $\mu$ and the coincidence set $\psi=\psi\left(x_{0}\right)$. In fact, it is easy to check that $\mu=0$ in the open set $\left\{\psi>\psi\left(x_{0}\right)\right\} \cap B_{\varepsilon}\left(x_{0}\right)$ in the sense that $\mu\left(\left\{\psi>\psi\left(x_{0}\right)\right\} \cap B_{\varepsilon}\left(x_{0}\right)\right)=0$. We thus deduce using the continuity of $\psi$ that

$$
\operatorname{supp}(\mu) \cap B_{\varepsilon}\left(x_{0}\right) \subset\left\{\psi=\psi\left(x_{0}\right)\right\} \cap B_{\varepsilon}\left(x_{0}\right) .
$$

But it is not obvious that these two sets should be equal. Nevertheless, we shall later see that, under a non-degeneracy condition on $F$, they are equal up to a set of measure zero. 
3.2. Regularity of $\psi$ and $\mu$. Proposition 3.3 will enable us to use classical regularity results for the obstacle problem to study the properties of $\varepsilon$-minimizers of $E$. Our first result is the following:

Theorem 3.4 ( $L^{\infty}$ regularity of $\left.\mu\right)$. Assume $W$ satisfies $(\mathrm{H} 1)$ and $(\mathrm{H} 2)$. Let $\mu$ be a compactly supported $\varepsilon$-minimizer in the sense of Definition 2.1. Assume moreover that one of the followings hold: either

(i) (H2) with $p>N$,

or

(ii) (H2) with $\frac{N}{2}<p \leq N$ and $\Delta W_{a} \in W_{\text {loc }}^{\varepsilon, 1}\left(\mathbb{R}^{N}\right)$ for some small $\varepsilon>0$.

Then the potential function $\psi$ is in $\mathcal{C}^{1,1}\left(\mathbb{R}^{N}\right)$. In particular, the measure $\mu$ is absolutely continuous with respect to the Lebesgue measure and there exists a function $\rho \in$ $L^{\infty}\left(\mathbb{R}^{N}\right)$ such that $\mu=\rho(x) d \mathcal{L}^{N}$. Finally, we have $\rho=\Delta W_{a} * \rho$ in the interior of $\operatorname{supp}(\mu)$.

Note that assumption (ii) requires slightly more regularity, but a lot less integrability that (i). It is satisfied by interaction potentials of the form $|x|^{q} / q$ as soon as $q>2-N$. The proof of this proposition will be given in Sect. 5 .

Remark 3.5. Under the conditions of Theorem 3.4, we can show that local minimizers are actually stationary states of the aggregation Eq. (2.2). Indeed, since $\nabla \psi \in C^{0,1}\left(\mathbb{R}^{N}\right)$ and $\rho \in L^{\infty}\left(\mathbb{R}^{N}\right)$ we have $\rho \nabla \psi \in L^{\infty}\left(\mathbb{R}^{N}\right)$. Moreover, since $\nabla \psi=0$ in the interior of $\operatorname{supp}(\rho)$, then $\rho \nabla \psi=0$ a.e. in $\mathbb{R}^{N}$, and thus $\rho$ is a classical stationary solution to (2.2).

Since $\Delta W_{a} \in L_{l o c}^{p}\left(\mathbb{R}^{N}\right)$ and $\rho \in L^{\infty}\left(\mathbb{R}^{N}\right)$ with compact support, a classical result for the convolution of functions implies that $\Delta W_{a} * \rho$ is a continuous function in $\mathbb{R}^{N}$. In particular, we deduce that $\rho$ is continuous in the interior of $\operatorname{supp}(\mu)$. But, in general, we will have $\Delta W_{a} * \rho \neq 0$ on $\partial(\operatorname{supp}(\mu))$ and so we expect $\rho$ to be discontinuous in $\mathbb{R}^{N}$. As a consequence, we cannot readily obtain further regularity for $\rho$ without assuming more regularity for $W_{a}$.

Obviously, if $W_{a}$ is smooth in $\mathbb{R}^{N}$, then $\rho$ will be smooth in the interior of $\operatorname{supp}(\mu)$. But it is not very difficult to prove (using a bootstrapping argument) that if $\Delta W_{a}$ is smooth in $\mathbb{R}^{N} \backslash\{0\}$ (as is the case for power like interaction potentials), then $\rho$ will be smooth in the interior of $\operatorname{supp}(\mu)$.

Finally, under the assumptions of Theorem 3.4, we note that since $\psi \in W^{2, \infty}$, we have

$$
\Delta \psi=-\rho+\Delta W_{a} * \rho=0 \quad \text { a.e. in }\left\{\psi=\psi\left(x_{0}\right)\right\} .
$$

If we assume further that $\Delta W_{a} * \rho>0$ in $B_{\varepsilon}\left(x_{0}\right)$, then we have $\rho(x)>0$ a.e. in $\left\{\psi=\psi\left(x_{0}\right)\right\}$ and thus

$$
\text { meas }\left(\left\{\psi=\psi\left(x_{0}\right)\right\} \cap B_{\varepsilon}\left(x_{0}\right) \backslash \operatorname{supp}(\mu)\right)=0,
$$

in other words, the support of $\mu$ and the coincidence set $\left\{\psi=\psi\left(x_{0}\right)\right\}$ are the same up to a set of measure zero.

As noted above, $\rho$ is expected to be a discontinuous function and so does not belong, in general, to $W_{l o c}^{1,1}$. However, under appropriate regularity assumption on $\Delta W_{a}$, we can prove that $\rho$ is in $\mathrm{BV}_{\text {loc }}\left(\mathbb{R}^{N}\right)$ : 
Theorem 3.6 (Regularity of supp $(\mu)$ ). Under the assumptions of Theorem 3.4, assume further that

$$
\Delta W_{a} \in W_{l o c}^{1,1}\left(\mathbb{R}^{N}\right) .
$$

Then the density $\rho$ lies in $\mathrm{BV}_{\text {loc }}\left(\mathbb{R}^{N}\right)$. Furthermore, if $\Delta W_{a} * \rho>0$ in a neighborhood of $\partial(\operatorname{supp}(\mu))$, then $\operatorname{supp}(\mu)$ is a set with locally finite perimeter.

Note that the condition that $\Delta W_{a} * \rho>0$ in a neighborhood of $\partial(\operatorname{supp}(\mu))$ is in particular satisfied if $\Delta W_{a}(x)$ is non-negative for all $x$ and not identically zero (which is the case when $W_{a}(x)=|x|^{q} / q$ with $\left.q>2-N\right)$. This condition implies that $\rho$ has a nonzero continuous extension on $\partial(\operatorname{supp}(\mu))$ from the interior of $\operatorname{supp}(\mu)$. In particular, $\rho$ has a jump discontinuity at the boundary of its support, and the $B V$ regularity is thus optimal in that sense.

Finally, let us point out that there are numerous results in the literature concerning further regularity of the free boundary $\partial(\operatorname{supp}(\mu))$ for the obstacle problem, always under the same non-degeneracy requirement that $\Delta W_{a} * \mu>0$ in a neighborhood of the free boundary, see $[8,10]$. Clearly many of these results could be used here, but we will not pursue this direction, as we are mainly interested in the regularity of the measure $\mu$ itself.

3.3. More singular than Newtonian case. Many of the results stated in the previous subsections can be extended without too much difficulty to the case in which the repulsion at zero in more singular than Newtonian but still locally integrable, that is, when

$$
W(x) \sim \frac{1}{|x|^{p}} \quad \text { as }|x| \rightarrow 0, \text { with } p \in(N-2, N)
$$

The general approach is the same as that described in the previous section, but the obstacle problem (3.7) will be replaced by a fractional obstacle problem of order less than 2 . Because such fractional obstacle problem enjoys very good properties, we will be able to derive regularity results for $\varepsilon$-minimizers.

To make this more precise, we first recall that $(-\Delta)^{s}$ denotes the fractional Laplace operator of order $s \in(0,1)$, which can be defined as a singular integral operator, or, using Fourier transform, as the operator with symbol $|\xi|^{2 s}$. For $s \in(0,1)$, it is then well known that the function

$$
V_{s}(x)=\frac{c_{N, s}}{|x|^{N-2 s}}
$$

is the fundamental solution of the fractional Laplace equation (for an appropriate choice of the constant $\left.c_{N, s}\right)$. More precisely, it satisfies $(-\Delta)^{s} V_{s}=\delta_{0}$. In this section, we fix $s \in(0,1)$ and consider an interaction potential that satisfies $W(x) \sim V_{s}(x)$ for $s \rightarrow 0$, that is, we replace hypothesis $(\mathrm{H} 2)$ with:

(H2s) The function $W_{a}(x):=W(x)-V_{s}(x)$ satisfies:

$$
\begin{aligned}
& (-\Delta)^{s} W_{a} \in L_{l o c}^{p}\left(\mathbb{R}^{N}\right) \text { for some } p \in\left(\frac{N}{2 s}, \infty\right] . \\
& (-\Delta)^{s} W_{a}(x) \leq C_{*} \text { a.e. } x \in \mathbb{R}^{N} .
\end{aligned}
$$

As before, we also define $W(0)=+\infty$. 
Finally, because of the non-locality of the fractional laplacian, it is much more difficult to deal with non compactly supported minimizers in this framework. For the sake of simplicity, and because the minimizers of interest in most applications have this property, we will thus only consider compactly supported local minimizers in this section (so (H3a) holds).

Remark 3.7. We note that when considering power-laws interaction potentials $W_{a}(x)=$ $\frac{|x|^{q}}{q}$, we get

$$
(-\Delta)^{s} W_{a}=|x|^{q-2 s} \frac{C_{N, s}}{q} \text { P.V. } \int_{\mathbb{R}^{N}} \frac{1-|z|^{q}}{|1-z|^{N+2 s}} d z,
$$

where we used the singular integral formulation of the fractional Laplacian. In particular, we need $q<2 s$ in order for the integral to be convergent and $q>0$ in order for (3.10) to hold. However, the restriction $q<2 s$ can be eliminated by truncating the potential outside a large ball.

As in the Newtonian case, the first step is to establish the continuity of the potential $\psi$ :

Proposition 3.8 (Continuity of the potential). Assume that the interaction potential $W$ satisfies (H1) and (H2s), and let $\mu$ be an $\varepsilon$-minimizer in the sense of Definition 2.1 such that $\operatorname{supp}(\mu)$ is compact. Then the potential $\psi(x):=W * \mu(x)$ is a continuous function in $\mathbb{R}^{N}$.

As a consequence, we can show (proceeding as in previous subsections):

Proposition 3.9. Under the conditions of Proposition 3.8, for all $x_{0} \in \operatorname{supp}(\mu)$, the potential function $\psi$ is equal, in $B_{\varepsilon}\left(x_{0}\right)$, to the unique solution of the obstacle problem

$$
\left\{\begin{aligned}
\varphi & \geq C_{0}, & & \text { in } B_{\varepsilon}\left(x_{0}\right) \\
(-\Delta)^{s} \varphi & \geq-F(x), & & \text { in } B_{\varepsilon}\left(x_{0}\right) \\
(-\Delta)^{s} \varphi & =-F(x), & & \text { in } B_{\varepsilon}\left(x_{0}\right) \cap\left\{\varphi>C_{0}\right\} \\
\varphi & =\psi, & & \text { in } \mathbb{R}^{N} \backslash B_{\varepsilon}\left(x_{0}\right),
\end{aligned}\right.
$$

where $C_{0}=\psi\left(x_{0}\right)$ and $F(x)=-(-\Delta)^{s} W_{a} * \mu \in L_{l o c}^{p}\left(\mathbb{R}^{N}\right)$. Furthermore, the density $\mu$ is given by

$$
\mu=(-\Delta)^{s} \psi+F .
$$

This type of obstacle problem has been studied by numerous authors in recent years, in particular by Silvestre [51]. However, some aspects of the theory for this fractional obstacle problem are different, or not as developed yet, as that of the regular obstacle problem. The only regularity result we will prove is that the density $\mu$ is Hölder continuous:

Theorem 3.10 (Regularity of $\mu$ ). Assume that $W$ satisfies $(\mathrm{H} 1)$ and $(\mathrm{H} 2 \mathrm{~s})$, and let $\mu$ be an $\varepsilon$-minimizer in the sense of Definition 2.1 such that $\operatorname{supp}(\mu)$ is compact. Assume moreover that

$$
(-\Delta)^{s} W_{a} \in W_{l o c}^{\varepsilon_{0}, 1}\left(\mathbb{R}^{N}\right) \text { for some small } \varepsilon_{0}>0 .
$$

Then the potential function $\psi$ is in $\mathcal{C}^{1, \gamma}\left(\mathbb{R}^{N}\right)$ for all $\gamma<s$. Furthermore, the measure $\mu$ is absolutely continuous with respect to the Lebesgue measure and there exists a function $\rho \in C^{\alpha}\left(\mathbb{R}^{N}\right)$ for all $\alpha<1-s$, such that $\mu=\rho(x) d \mathcal{L}^{N}$. 
Remark 3.11. The optimal regularity for the potential function in the fractional obstacle problem is $C^{1, s}\left(\mathbb{R}^{N}\right)$, see [14], but it requires $W_{a} * \mu \in C^{2,1}\left(\mathbb{R}^{N}\right)$.

Note that it has been conjectured that the free boundary has locally finite $N-1$ Hausdorff measure for the fractional obstacle problem, but to our knowledge there is no proof of it yet.

3.4. A uniqueness result. We end this section with a uniqueness result for $d_{2}$-local minimizers for the very particular case of quadratic confinement where $W_{a}(x)=K|x|^{2}$. This result gives an alternative proof of the classical results by potential theory mentioned in the introduction [26,34,41], see also [27].

Let us remark that the results in [17] show the existence of global minimizers for $W(x)=K|x|^{2}+V_{s}(x)$, see [17, Sect. 3]. Moreover, all global minimizers must be compactly supported and an attentive reading of Lemmas 2.6 and 2.7 in [17] shows that any $d_{2}$-local minimizer is compactly supported in this particular case, since $W(x) \rightarrow \infty$ as $|x| \rightarrow \infty$. Our result is:

Theorem 3.12 (Uniqueness of $d_{2}$ minimizer). Assume that $W_{a}(x)=K|x|^{2}$, where $K$ is a constant, and that $(\mathrm{H} 1)$ and either $(\mathrm{H} 2)$ or $(\mathrm{H} 2 \mathrm{~s})$ hold. Then there exists a unique (up to translation) $d_{2}$-local minimizer $\mu_{0} \in \mathcal{P}_{2}\left(\mathbb{R}^{N}\right)$, which is also the unique global minimizer of $E$ in $\mathcal{P}_{2}\left(\mathbb{R}^{N}\right)$. Furthermore, $\mu_{0}$ is compactly supported and radial symmetric.

As discussed in Sect. 2, for $d_{2}$-local minimizers, we have

$$
\psi(x)=2 E[\mu] \text { for all } x \in \operatorname{supp}(\mu) .
$$

In other words, the constant $C_{0}$ appearing in Proposition 3.3 does not depend on the choice of $x_{0}$. We will thus prove Theorem 3.12 by using a uniqueness result for the obstacle problem, in the particular case treated in [15].

In the case of Newtonian repulsion (condition (H2)), we get a slightly better result. Indeed, for this choice of $W_{a}$, we have $\Delta W_{a}=2 N K$, so by Eq. (3.8), we have

$$
\mu(x)=F(x)=2 N K \quad \text { for all } x \in \operatorname{supp}(\mu) .
$$

In other words, the regularity that we have proven in the previous sections allows us to say that $\mu$ has to be a multiple of the characteristic function of its support, and it is not difficult to show that this support is a ball.

We can also use this information a priori and obtain a different proof of Theorem 3.12 for global minimizers in the Newtonian case using a simple scaling argument and a trivial rearrangement. This proof uses the obstacle problem to allow us to assume that $\mu$ is a function satisfying (3.12). We provide this proof for the interested reader at the end of Sect. 7.

\section{Continuity of the Potential Function $\psi$}

This section contains the proof of Proposition 3.2. First, we recall that for a given $\mu \in \mathcal{P}\left(\mathbb{R}^{N}\right)$ and due to (H1), the potential function $\psi=W * \mu$, is defined pointwise in $\mathbb{R}^{N}$, with values in $[0, \infty]$, by

$$
\psi(x):=W * \mu=\int_{\mathbb{R}^{N}} W(x-y) d \mu(y) .
$$


Furthermore, $\psi$ is lower semicontinuous. We also recall that whether $(\mathrm{H} 3 \mathrm{a})$ or $(\mathrm{H} 3 \mathrm{~b})$ holds, we can always assume (3.4), i.e., that there exists a constant $M$ such that

$$
\left\|\Delta W_{a}\right\|_{L^{p}\left(B_{1}(x)\right)} \leq M \text { for all } x \in \mathbb{R}^{N} .
$$

The proof of Proposition 3.2 will rely on the following classical mean value formula, whose proof is recalled in Appendix A for the reader's sake.

Lemma 4.1. Let $u$ be such that $\Delta u \in L_{\text {loc }}^{p}\left(\mathbb{R}^{N}\right)$ for some $p \in\left(\frac{N}{2}, \infty\right]$. Then for all $x$, there exists a constant $C$ depending only on $N$ and $p$, such that

$$
u(x) \geq \frac{1}{\left|B_{r}\right|} \int_{B_{r}(x)} u(y) d y-C\|\Delta u\|_{L^{p}\left(B_{1}(x)\right)} r^{\alpha}, \quad \text { for all } r \in(0,1),
$$

with $\alpha=2-\frac{N}{p}, N \geq 3$. When $N=2, r^{\alpha}$ is replaced by $|\log r| r^{\alpha}$.

As a simple application of this result, we can show

Lemma 4.2. Let $\mu \in \mathcal{P}\left(\mathbb{R}^{N}\right)$ and assume (H1), (H2), and (H3). Then there exists a constant $C$ depending on $N, p$, and the constant $M$ appearing in (3.4), such that the potential function $\psi=W * \mu$ satisfies

$$
\psi(x) \geq \frac{1}{\left|B_{r}\right|} \int_{B_{r}(x)} \psi(y) d y-C r^{\alpha}
$$

for all $x \in \mathbb{R}^{N}$ and all $r \in(0,1)$. When $N=2, r^{\alpha}$ is replaced by $|\log r| r^{\alpha}$.

Proof of Lemma 4.2. Observe that we can always assume $\psi(x)<\infty$, since otherwise (4.1) clearly holds. Next, we recall that $W=V+W_{a}$ where the function $V$ is superharmonic, and thus satisfies

$$
V(x-y) \geq \frac{1}{\left|B_{r}\right|} \int_{B_{r}(0)} V(x-y+z) d z
$$

for all $x$ and $y \in \mathbb{R}^{N}$. Furthermore, the function $W_{a}$ satisfies the condition of Lemma 4.1 due to $(\mathrm{H} 2)$, and so

$$
W(x-y) \geq \frac{1}{\left|B_{r}\right|} \int_{B_{r}(0)} W(x-y+z) d z-C r^{\alpha}
$$

for all $r \in(0,1)$, where the constant $C$ depends on $M$. We deduce

$$
\psi(x)=\int_{\mathbb{R}^{N}} W(x-y) d \mu(y) \geq \frac{1}{\left|B_{r}\right|} \int_{\mathbb{R}^{N}} \int_{B_{r}(0)} W(x-y+z) d z d \mu(y)-C r^{\alpha},
$$

and Fubini-Tonelli theorem implies

$$
\begin{aligned}
\int_{\mathbb{R}^{N}} \int_{B_{r}(0)} W(x-y+z) d z d \mu(y) & =\int_{B_{r}(0)} \int_{\mathbb{R}^{N}} W(x-y+z) d \mu(y) d z \\
& =\int_{B_{r}(x)} \psi(z) d z .
\end{aligned}
$$

Notice that the integral in the left hand side is finite thanks to (H1). The result follows and the $N=2$ case is totally analogous. 
With the previous Lemma, we can prove the following Corollary that gives important information about the potential function in the support of the local minimizer.

Corollary 4.3. Let $\mu$ be an $\varepsilon$-minimizer of the energy $E$ in the sense of Definition 2.1 and assume that (H1), (H2), and (H3) hold. Then any point $x_{0} \in \operatorname{supp}(\mu)$ is a local minimizer of $\psi=W * \mu$ in the sense that

$$
\psi\left(x_{0}\right) \leq \psi(x) \text { for all } x \in B_{\varepsilon}\left(x_{0}\right) .
$$

Furthermore, we have

$$
\psi(x)=\psi\left(x_{0}\right) \quad \text { for all } x \in \operatorname{supp}(\mu) \cap B_{\varepsilon}\left(x_{0}\right) .
$$

Proof of Corollary 4.3. Note that (4.1) implies

$$
\psi(x) \geq \lim _{r \rightarrow 0} \frac{1}{\left|B_{r}\right|} \int_{B_{r}(x)} \psi(y) d y \quad \text { for all } x \in B_{\varepsilon}\left(x_{0}\right) .
$$

But for any $x \in B_{\epsilon}\left(x_{0}\right), B_{r}(x) \subset B_{\epsilon}\left(x_{0}\right)$ for small enough $r>0$. Proposition 2.2 implies that

$$
\psi(y) \geq \psi\left(x_{0}\right) \quad \text { a.e. } y \in B_{r}(x) .
$$

So from (4.3), we conclude that

$$
\psi(x) \geq \psi\left(x_{0}\right) \quad \text { for all } x \in B_{\epsilon}(x) .
$$

Because the $\varepsilon$ does not depend on the choice of $x_{0}$ by Remark 2.3, (4.2) easily follows.

Using the previous Corollary we can prove (as a first step toward the continuity of the potential function $\psi$ ) that $W_{a} * \mu$ is continuous.

Lemma 4.4. Let $\mu$ be an $\varepsilon$-minimizer of the energy $E$ in the sense of Definition 2.1 and assume that (H1), (H2), and either (H3a) or (H3b) hold. Then, $\psi \in L_{\text {loc }}^{\infty}\left(\mathbb{R}^{N}\right)$ and $W_{a} * \mu \in C_{\text {loc }}^{\alpha}\left(\mathbb{R}^{N}\right)$, where $\alpha=2-\frac{N}{p}$.

Proof. Condition (H2) implies that $W_{a} \in C_{l o c}^{\alpha}$, so under (H3a), the result is obvious. We can thus assume that only (H3b) holds. The difficulty in that case is to deal with the large values of $|x|$ in the support of $\mu$.

First, we prove that $\psi$ is finite everywhere in the support of $\mu$. Clearly, we have $\psi(x) \geq 0$ (since $W \geq 0$ ). Furthermore, given $y_{0} \in \operatorname{supp}(\mu)$, using Corollary 4.3 we know that $\psi(x) \geq \psi\left(y_{0}\right)$ in $B_{\varepsilon}\left(y_{0}\right)$ and we can write:

$$
E[\mu]=\int_{\mathbb{R}^{N}} \psi(x) d \mu(x) \geq \int_{B_{\varepsilon}\left(y_{0}\right)} \psi(x) d \mu(x) \geq \mu\left(B_{\varepsilon}\left(y_{0}\right)\right) \psi\left(y_{0}\right) .
$$

Since $y_{0} \in \operatorname{supp}(\mu)$, we have $\mu\left(B_{\varepsilon}\left(y_{0}\right)\right)>0$ and so

$$
\psi\left(y_{0}\right) \leq \frac{E[\mu]}{\mu\left(B_{\varepsilon}\left(y_{0}\right)\right)}<\infty .
$$

Now, given $x_{0} \in \mathbb{R}^{N} \backslash \operatorname{supp}(\mu)$, let $R=d\left(x_{0}, \operatorname{supp}(\mu)\right)$, then by (H2) we know that

$$
\Delta \psi=\Delta W_{a} * \mu \geq-C_{*} \quad \text { in } B_{R}\left(x_{0}\right) .
$$


Therefore, by applying the mean-value formula to the subharmonic function $\psi+$ $C_{*} \frac{\left|x-x_{0}\right|^{2}}{2 N}$, we obtain

$$
\psi\left(x_{0}\right) \leq \frac{1}{\left|B_{R}\right|} \int_{B_{R}\left(x_{0}\right)} \psi(y) d y+\frac{C_{*}}{2 N} R^{2} .
$$

Let now $y_{0}$ be the closest point in $\operatorname{supp}(\mu)$ to $x_{0}$. Then proceeding as for Lemma 4.2, we obtain

$$
\psi\left(y_{0}\right) \geq \frac{1}{\left|B_{2 R}\right|} \int_{B_{2 R}\left(x_{1}\right)} \psi(y) d y-C(R) .
$$

Combining both inequalities we deduce

$$
\psi\left(x_{0}\right) \leq C\left(d\left(x_{0}, \operatorname{supp}(\mu)\right), \psi\left(y_{0}\right)\right)<\infty .
$$

We now want to show that $\psi$ is bounded in $B_{1}\left(x_{0}\right)$. First, assume that $\operatorname{supp}(\mu) \cap$ $B_{2}\left(x_{0}\right)=\varnothing$. Then, for all $x \in B_{1}\left(x_{0}\right)$, we have

$$
\Delta \psi=\Delta W_{a} * \mu \geq-C_{*} \quad \text { in } B_{1}(x)
$$

and proceeding as above, we deduce

$$
\psi(x) \leq \frac{1}{\left|B_{1}\right|} \int_{B_{1}(x)} \psi(y) d y+\frac{C_{*}}{2 N} \leq \frac{2}{\left|B_{2}\right|} \int_{B_{2}\left(x_{0}\right)} \psi(y) d y+\frac{C_{*}}{2 N}
$$

and we conclude (using Lemma 4.2) that

$$
\psi(x) \leq C\left(\psi\left(x_{0}\right)\right) \quad \text { in } B_{1}\left(x_{0}\right) .
$$

If $\operatorname{supp}(\mu) \cap B_{2}\left(x_{0}\right) \neq \emptyset$, we note that $\operatorname{supp}(\mu) \cap B_{2}\left(x_{0}\right)$ can be covered by a finite number of balls of size $\varepsilon$ :

$$
\operatorname{supp}(\mu) \cap B_{2}\left(x_{0}\right) \subset \cup_{i=1}^{p} B_{\varepsilon}\left(y_{i}\right)
$$

with $\mu\left(B_{\varepsilon}\left(y_{i}\right)\right)>0$ for all $i$. Since $\psi$ is constant in $\operatorname{supp}(\mu) \cap B_{\varepsilon}\left(y_{i}\right)$, (4.4) implies that there exists a constant $C$ (depending on $x_{0}$ of course) such that

$$
\psi(y) \leq C \quad \text { for all } y \in \operatorname{supp}(\mu) \cap B_{2}\left(x_{0}\right) .
$$

Now, for a given $x \in B_{1}\left(x_{0}\right)$, either $B_{1}(x) \cap \operatorname{supp}(\mu)=\emptyset$ in which case we get (4.6), or $d(x, \operatorname{supp}(\mu))<1$, in which case we conclude using (4.5) and (4.7).

This concludes the proof of the fact that $\psi$ is in $L_{l o c}^{\infty}\left(\mathbb{R}^{N}\right)$.

To prove the Hölder continuity of $W_{a} * \mu$, we take $x$ and $y$ such that $|x-y| \leq 1$, then

$$
\left|W_{a}(x-z)-W_{a}(y-z)\right| \leq|| W_{a} \|_{C^{\alpha}\left(B_{1}(x-z)\right)}|x-y|^{\alpha}
$$

for all $x, z \in \mathbb{R}^{N}$, and using Sobolev embeddings with $\alpha=2-\frac{N}{p}$, we get the bound

$$
\begin{aligned}
\left\|W_{a}\right\|_{C^{\alpha}\left(B_{1}(x-z)\right)} & \leq \int_{B_{1}(x)}\left|W_{a}(t-z)\right| d t+\left\|\Delta W_{a}\right\|_{L^{p}\left(B_{1}(x-z)\right)} \\
& \leq \int_{B_{1}(x)}\left|W_{a}(t-z)\right| d t+M
\end{aligned}
$$


for all $x, z \in \mathbb{R}^{N}$ (where $\mathrm{M}$ is the uniform bound from (H3b)). We thus have

$$
\begin{array}{rl}
\mid W_{a} & * \mu(x)-W_{a} * \mu(y) \mid \\
& \leq \int_{\mathbb{R}^{N}}\left|W_{a}(x-z)-W_{a}(y-z)\right| d \mu(z) \\
& \leq \int_{\mathbb{R}^{N}}\left(\int_{B_{1}(x)}\left|W_{a}(t-z)\right| d t+M\right)|x-y|^{\alpha} d \mu(z) \\
& \leq\left(\int_{B_{1}(x)} \int_{R^{N}}\left(W_{a}(t-z)-2 \inf W_{a}\right) d \mu(z) d t+M\right)|x-y|^{\alpha} .
\end{array}
$$

In dimension $N \geq 3$, using the fact that $V(x) \geq 0$, we deduce

$$
\left|W_{a} * \mu(x)-W_{a} * \mu(y)\right| \leq\left(2 C\left|\inf W_{a}\right|+\int_{B_{1}(x)} \psi(t) d t+M\right)|x-y|^{\alpha},
$$

and in dimension $N=2$, we get

$$
\begin{aligned}
& \left|W_{a} * \mu(x)-W_{a} * \mu(y)\right| \\
& \quad \leq\left(2 C\left|\inf W_{a}\right|+\int_{B_{1}(x)} \psi(t) d t+C \int_{B_{1}(x)} \int_{\mathbb{R}^{N}} \log _{+}(|t-y|) d \mu(y) d t+M\right)|x-y|^{\alpha} \\
& \quad \leq\left(2 C\left|\inf W_{a}\right|+\int_{B_{1}(x)} \psi(t) d t+C \int_{\mathbb{R}^{N}} \log _{+}(1+|y|) d \mu(y)+M\right)|x-y|^{\alpha} .
\end{aligned}
$$

We can now conclude using the fact that $\psi$ is in $L_{l o c}^{\infty}$ (and (3.5) when $N=2$ ).

We now have everything that we need in order to prove Proposition 3.2 by reproducing Evans's proof for the continuity of the solution of the obstacle problem (see [10] for instance):

Proof of Proposition 3.2. Corollary 4.3 implies that $\psi$ is continuous in the support of $\mu$ in the following sense: If $x_{k} \in \operatorname{supp}(\mu)$ and $x_{k} \rightarrow x_{0} \in \operatorname{supp}(\mu)$, then $\psi\left(x_{k}\right) \longrightarrow \psi\left(x_{0}\right)$.

Assume now that we have a sequence $x_{k} \in \mathbb{R}^{N}$ such that $x_{k} \rightarrow x_{0} \notin \operatorname{supp}(\mu)$. By definition of $\operatorname{supp}(\mu)$, there exists a ball $B_{r}\left(x_{0}\right)$ such that $\mu\left(B_{r}\left(x_{0}\right)\right)=0$. In particular, the function $\psi-W_{a} * \mu$ is harmonic, and thus continuous, in $B_{r / 2}\left(x_{0}\right)$. Furthermore, Lemma 4.4 implies that $W_{a} * \mu$ is continuous in $B_{r / 2}\left(x_{0}\right)$, so $\psi$ is continuous in $B_{r / 2}\left(x_{0}\right)$, and $\lim _{k \rightarrow \infty} \psi\left(x_{k}\right)=\psi\left(x_{0}\right)$.

We now assume that $x_{0} \in \operatorname{supp}(\mu)$ and consider a sequence $x_{k} \in \mathbb{R}^{N}$ such that $x_{k} \rightarrow x_{0}$ as $k \rightarrow \infty$. We can always assume that $x_{k} \in B_{\varepsilon}\left(x_{0}\right)$ for all $k$. In particular, if $x_{k} \in \operatorname{supp}(\mu)$ then (4.2) implies $\psi\left(x_{k}\right)=\psi\left(x_{0}\right)$. So we can consider a subsequence (still denoted $x_{k}$ ) such that

$$
x_{k} \notin \operatorname{supp}(\mu) \text { for all } k, \quad \lim _{k \rightarrow \infty} x_{k}=x_{0} \in \operatorname{supp}(\mu) .
$$

This is of course the main step in Evans's Theorem. Since $x_{k} \in B_{\varepsilon}\left(x_{0}\right)$ for all $k,(2.3)$ implies

$$
\psi\left(x_{k}\right) \geq \psi\left(x_{0}\right) \quad \text { for all } k .
$$


Let $y_{k}$ be the closest point in $\operatorname{supp}(\mu) \cap \overline{B_{\varepsilon / 2}}\left(x_{0}\right)$ to $x_{k}$. So (4.2) implies that $\psi\left(y_{k}\right)=$ $\psi\left(x_{0}\right)$. We denote $\delta_{k}=\left|x_{k}-y_{k}\right|$. Notice that $\delta_{k}$ is the distance of $x_{k}$ to $\operatorname{supp}(\mu)$, and so $\delta_{k} \leq\left|x_{k}-x_{0}\right| \rightarrow 0$. Inequality (4.1) implies

$$
\psi\left(x_{0}\right)=\psi\left(y_{k}\right) \geq \frac{1}{\left|B_{2 \delta_{k}}\left(y_{k}\right)\right|} \int_{B_{2 \delta_{k}}\left(y_{k}\right)} \psi(y) d y-C \delta_{k}^{\alpha},
$$

and so

$$
\frac{1}{\left|B_{2 \delta_{k}}\left(y_{k}\right)\right|} \int_{B_{2 \delta_{k}}\left(y_{k}\right)}\left[\psi(y)-\psi\left(x_{0}\right)\right] d y \leq C \delta_{k}^{\alpha} .
$$

Since $\psi(y)-\psi\left(x_{0}\right) \geq 0$ in $B_{2 \delta_{k}}\left(y_{k}\right)$ (for $k$ large enough) and $B_{\delta_{k} / 2}\left(x_{k}\right) \subset B_{2 \delta_{k}}\left(y_{k}\right)$, we deduce

$$
\frac{1}{4^{d}\left|B_{\delta_{k} / 2}\left(x_{k}\right)\right|} \int_{B_{\delta_{k} / 2}\left(x_{k}\right)}\left[\psi(y)-\psi\left(x_{0}\right)\right] d y \leq C \delta_{k}^{\alpha} .
$$

Furthermore, since $B_{\delta_{k} / 2}\left(x_{k}\right)$ is away from $\operatorname{supp}(\mu)$, and $\Delta V(x)=0$ for all $x \neq 0$, we deduce by (3.3) in (H2) that

$$
\Delta \psi=\Delta W_{a} * \mu \geq-C_{*} \quad \text { in } B_{\delta_{k} / 2}\left(x_{k}\right) .
$$

Since the function $\psi+C_{*} \frac{\left|x-x_{k}\right|^{2}}{2 N}$ is subharmonic, we deduce that

$$
\psi\left(x_{k}\right) \leq \frac{1}{\left|B_{\delta_{k} / 2}\right|} \int_{B_{\delta_{k} / 2}\left(x_{k}\right)} \psi(y) d y+\frac{C_{*}}{2 N} \delta_{k}^{2}
$$

and so

$$
\psi\left(x_{k}\right)-\psi\left(x_{0}\right) \leq \frac{1}{\left|B_{\delta_{k} / 2}\right|} \int_{B_{\delta_{k} / 2}\left(x_{k}\right)}\left[\psi(y)-\psi\left(x_{0}\right)\right] d y+\frac{C_{*}}{2 N} \delta_{k}^{2} \leq 4^{d} \delta_{k}^{\alpha}+\frac{C_{*}}{2 N} \delta_{k}^{2} .
$$

Together with (4.8), this implies

$$
\lim _{k \rightarrow \infty} \psi\left(x_{k}\right)=\psi\left(x_{0}\right),
$$

and the result of Proposition 3.2 follows.

\section{Regularity of $\mu$}

In this section, we prove the regularity results for $\psi$ and $\mu$ stated in Theorems 3.4 and 3.6. For that, we are going back and forth between the regularity of the solution of the obstacle problem (3.7) and the regularity of $F=\Delta W_{a} * \mu$. To begin with, we note that Minkowski integral's inequality [53, A.1] and (H3) implies

$$
\begin{aligned}
\left(\int_{B_{1}\left(x_{0}\right)}|F(x)|^{p} d x\right)^{1 / p} & =\left(\int_{B_{1}\left(x_{0}\right)}\left|\int_{\mathbb{R}^{N}} \Delta W_{a}(x-y) d \mu(y)\right|^{p} d x\right)^{1 / p} \\
& \leq \int_{\mathbb{R}^{N}}\left(\int_{B_{1}\left(x_{0}\right)}\left|\Delta W_{a}(x-y)\right|^{p} d x\right)^{1 / p} d \mu(y) \\
& \leq M
\end{aligned}
$$

and so $F$ is locally uniformly in $L^{p}$. 
5.1. Proof of Theorem $3.4(i)$. If we now assume that $(\mathrm{H} 2)$ holds with $p>N$. Then, for all $x_{0} \in \operatorname{supp}(\mu)$, we have $F \in L^{p}\left(B_{\varepsilon}\left(x_{0}\right)\right)$ with $p>N$, and it is a classical result [36] that the solution of the obstacle problem (3.7) is such that $\Delta \psi$ is a function and satisfies

$$
-F \leq-\Delta \psi \leq \max \{0,-F\} \quad \text { in } B_{\varepsilon}\left(x_{0}\right) .
$$

Since this is true for all $x_{0} \in \operatorname{supp}(\mu)$, we deduce that $\mu$ has density $\rho$ and

$$
0 \leq \rho \leq \max \{F, 0\} \quad \text { in } \mathbb{R}^{N}
$$

and so $\rho$ is locally uniformly in $L^{p}\left(\mathbb{R}^{N}\right)$. By assumption, we know that $\rho$ is compactly supported, then $\rho \in L^{p}\left(\mathbb{R}^{N}\right)$ and thus, $F=\Delta W_{a} * \rho \in C\left(\mathbb{R}^{N}\right)$ by standard convolution properties since $p>N \geq 2$. Using again (5.1) and the compact support of $\rho$, we deduce that $\rho \in L^{\infty}\left(\mathbb{R}^{N}\right)$. This concludes the proof of Theorem 3.4 when $(i)$ holds.

5.2. Proof of Theorem 3.4(ii). Unfortunately, when $p \leq N$ (which is the case for kernels of the form $|x|^{q} / q$ when $q \leq 1$ ), inequality (5.1) does not hold, and the argument above cannot be used. We are thus now going to prove the $L^{\infty}$ regularity of $\mu$ under Assumption (ii) of Theorem 3.4.

Since we are assuming that $\operatorname{supp}(\mu) \subset B_{R}(0)$, and we are only interested in the properties of $\psi$ and $\mu$ in a neighborhood of $\operatorname{supp}(\mu)$, it is possible to modify the values of $W_{a}$ outside a ball $B_{2 R}(0)$ without changing the values of $\mu$ and $\psi$ in $B_{R}$ as discussed in Sect. 3. We can thus assume that $\Delta W_{a}$ has compact support and that

$$
\Delta W_{a} \in W^{\epsilon, 1}\left(\mathbb{R}^{N}\right) \text { for some } \epsilon>0 .
$$

We will then use the following lemma (with $K=\Delta W_{a}$ ):

Lemma 5.1. If $K \in W^{\epsilon, 1}\left(\mathbb{R}^{N}\right)$ with compact support and $\varphi \in C^{\alpha}\left(\mathbb{R}^{N}\right)$ is a bounded function, then $K * \varphi \in C^{\alpha+\varepsilon}$.

Proof of Lemma 5.1. We note that

$$
(-\Delta)^{\varepsilon / 2}(K * \varphi)=\left[(-\Delta)^{\varepsilon / 2} K\right] * \varphi
$$

is the convolution of an $L^{1}\left(\mathbb{R}^{N}\right)$ function with a $\mathcal{C}^{\alpha}\left(\mathbb{R}^{N}\right)$ function. We thus have $(-\Delta)^{\varepsilon / 2}(K * \varphi) \in \mathcal{C}^{\alpha}\left(\mathbb{R}^{N}\right)$. Standard regularity result for fractional elliptic equation (see [51]) implies $K * \varphi \in \mathcal{C}^{\alpha+\varepsilon}\left(\mathbb{R}^{N}\right)$.

We will then rely on the following important result for the regularity of the solution of the obstacle problem in [10]:

Proposition 5.2. Let $\psi$ be the solution of the obstacle problem (3.7). Then up to $C^{1,1}\left(\mathbb{R}^{N}\right)$ the function $\psi$ is as regular as $W_{a} * \mu$. More precisely, we have

- If $W_{a} * \mu$ has a modulus of continuity $\sigma(r)$, then $\psi$ has a modulus of continuity $C \sigma(2 r)$.

- If $\nabla W_{a} * \mu$ has a modulus of continuity of $\sigma(r)$, then $\nabla \psi$ has a modulus of continuity $C \sigma(2 r)$.

Using a bootstrap argument and Lemma 5.1, we can now prove Theorem 3.4 when (ii) holds: 
Proof of Theorem 3.4 (ii). (H2) together with the compact support of $\Delta W_{a}$ imply that $\Delta W_{a} * \mu \in L^{p}\left(\mathbb{R}^{N}\right)$ and so $W_{a} * \mu \in W^{2, p}\left(\mathbb{R}^{N}\right)$ for some $p>N / 2$. In particular, we have

$$
W_{a} * \mu \in \mathcal{C}^{\alpha}\left(\mathbb{R}^{N}\right) \quad \text { with } \alpha=2-\frac{N}{p},
$$

and Proposition 5.2 implies

$$
\psi \in \mathcal{C}^{\alpha}\left(\mathbb{R}^{N}\right) \quad \text { with } \alpha=2-\frac{N}{p} .
$$

Since $\mu=-\Delta \psi+\Delta W_{a} * \mu$, we can write

$$
W_{a} * \mu=-W_{a} * \Delta \psi+W_{a} *\left(\Delta W_{a} * \mu\right)=-\Delta W_{a} *\left(\psi+W_{a} * \mu\right) .
$$

As $\Delta W_{a} \in W^{\varepsilon, 1}\left(\mathbb{R}^{N}\right)$, we can use Lemma 5.1 to show that

$$
W_{a} * \mu \in \mathcal{C}^{\alpha+\varepsilon}\left(\mathbb{R}^{N}\right) .
$$

A simple bootstrap argument yields that $W_{a} * \mu$ and $\psi$ are both in $\mathcal{C}^{1,1}\left(\mathbb{R}^{N}\right)$, and thus that $\mu$ has density $\rho \in L^{\infty}\left(\mathbb{R}^{N}\right)$.

5.3. BV estimate and measure theoretic regularity of the free boundary. We now prove Theorem 3.6. We note that under the assumption of Theorem 3.6, we have $F \in W_{l o c}^{1,1}\left(\mathbb{R}^{N}\right)$ and, for the second part of the statement, we get $F(x) \neq 0$ in a bounded open neighbor$\operatorname{hood}$ of $\operatorname{supp}(\mu)$.

Under these assumptions, the regularity in $\mathrm{BV}_{\text {loc }}\left(\mathbb{R}^{N}\right)$ of $\Delta \psi$, where $\psi$ solves the obstacle problem (3.7) is a classical result, which implies Theorem 3.6. We will sketch the proof of this result for the reader's sake. The proof that we give below was first proposed by Brezis and Kinderlehrer in [9].

Proof of Theorem 3.6. First, we recall that the solution of the obstacle problem (3.7) can be approximated by the solutions $\psi_{\delta}$ of the nonlinear equation

$$
\begin{aligned}
-\Delta \psi_{\delta}+\beta_{\delta}\left(\psi_{\delta}-C_{0}\right) & =-F \text { in } \Omega \\
\psi_{\delta} & =\psi \text { on } \partial \Omega
\end{aligned}
$$

where $\Omega=B_{\epsilon}\left(x_{0}\right)$ with $x_{0} \in \operatorname{supp}(\mu)$ and $\beta_{\delta}$ is an increasing function satisfying $s \beta_{\delta}(s) \geq 0$ for all $s$ and such that

$$
\beta_{\delta}(s) \longrightarrow\left\{\begin{array}{ll}
0 & \text { when } s>0 \\
-\infty & \text { when } s<0
\end{array} \quad \text { as } \delta \rightarrow 0 .\right.
$$

Here, $\Omega=B_{\varepsilon}\left(x_{0}\right)$ for any point $x_{0} \in \operatorname{supp}(\mu)$. It is a classical result, see [9] for instance, that $\psi_{\delta}$ converges to $\psi$ locally uniformly in $C^{1, \gamma}(\Omega)$ provided $F$ is in $L^{\infty}(\Omega)$ (which we proved in Theorem 3.4).

Let now $\frac{\partial}{\partial \xi}$ denote any directional derivative, we are going to show that for any compact set $K \subset \subset \Omega$, we have

$$
\int_{K}\left|\frac{\partial}{\partial x_{i}} \Delta \psi_{\delta}\right| \leq C
$$


where $C$ does not depend on $\delta$. Taking the limit $\delta \rightarrow 0$ and using the 1.s.c. of the total variation, we deduce that $\Delta \psi \in B V_{l o c}(\Omega)$, which gives the result.

In order to prove (5.3), we differentiate (5.2):

$$
-\Delta \partial_{\xi} \psi_{\delta}+\beta_{\delta}^{\prime}\left(\psi_{\delta}-C_{0}\right) \partial_{\xi} \psi_{\delta}=-\partial_{\xi} F
$$

Let now $\chi$ be a test function in $\mathcal{D}(\Omega)$ such that $\chi \geq 0$ in $\Omega$ and $\chi=1$ in $K$. We multiply (5.4) by $\chi \operatorname{sign}\left(\partial_{\xi} \psi_{\delta}\right)$ and integrate over $\Omega$ to deduce

$$
\begin{aligned}
& -\int_{\Omega} \chi \operatorname{sign}\left(\partial_{\xi} \psi_{\delta}\right) \Delta \partial_{\xi} \psi_{\delta} d x+\int_{\Omega} \beta_{\delta}^{\prime}\left(\psi_{\delta}-C_{0}\right)\left|\partial_{\xi} \psi_{\delta}\right| \chi d x \\
& =-\int_{\Omega} \partial_{\xi} F \chi \operatorname{sign}\left(\partial_{\xi} \psi_{\delta}\right) d x .
\end{aligned}
$$

Integrating by parts the left hand side yields

$$
\begin{aligned}
\int_{\Omega} \chi \operatorname{sign}^{\prime}\left(\partial_{\xi} \psi_{\delta}\right)\left|\nabla \frac{\partial}{\partial \xi} \psi_{\delta}\right|^{2} d x+\int_{\Omega} \beta_{\delta}^{\prime}\left(\psi_{\delta}-C_{0}\right)\left|\partial_{\xi} \psi_{\delta}\right| \chi d x \\
=-\int_{\Omega} \nabla \chi \nabla \partial_{\xi} \psi_{\delta} \operatorname{sign}\left(\partial_{\xi} \psi_{\delta}\right) d x-\int_{\Omega} \partial_{\xi} F \chi \operatorname{sign}\left(\partial_{\xi} \psi_{\delta}\right) d x .
\end{aligned}
$$

Using the fact that $\operatorname{sign}^{\prime}(s) \geq 0$ for all $s$, we deduce

$$
\begin{aligned}
\int_{\Omega} \beta_{\delta}^{\prime}\left(\psi_{\delta}-C_{0}\right)\left|\partial_{\xi} \psi_{\delta}\right| \chi d x & \leq-\int_{\Omega} \nabla \chi \nabla\left|\partial_{\xi} \psi_{\delta}\right| d x-\int_{\Omega} \partial_{\xi} F \chi \operatorname{sign}\left(\partial_{\xi} \psi_{\delta}\right) d x \\
& \leq \int_{\Omega} \Delta \chi\left|\partial_{\xi} \psi_{\delta}\right| d x+\int_{\Omega}\left|\partial_{\xi} F\right| \chi d x .
\end{aligned}
$$

Furthermore, multiplying (5.2) by $\left(\psi_{\delta}-C_{0}\right) \chi$, it is easy to show that

$$
\int_{K}\left|\partial_{\xi} \psi_{\delta}\right|^{2} d x \leq C(K)
$$

for some constant depending on $K$ but not on $\delta$ (using the regularity of $F$ and the fact that $\psi_{\delta}$ converges locally uniformly to $\left.\psi\right)$. We conclude that

$$
\int_{K} \beta_{\delta}^{\prime}\left(\psi_{\delta}-C_{0}\right)\left|\partial_{\xi} \psi_{\delta}\right| d x \leq C
$$

with $C$ independent of $\delta$. Finally, going back to (5.4), we get

$$
\int_{K}\left|\Delta \partial_{\xi} \psi_{\delta}\right| d x \leq \int_{K} \beta_{\delta}^{\prime}\left(\psi_{\delta}-C_{0}\right)\left|\partial_{\xi} \psi_{\delta}\right| d x+\int_{K}\left|\partial_{\xi} F\right| d x \leq C
$$

and the result follows.

To prove the second part of the Theorem, we note that the function

$$
\frac{-\Delta \psi+F}{F}=\frac{\rho}{F}
$$

is almost everywhere equal to the indicator function of the set $\left\{\psi=\psi\left(x_{0}\right)\right\} \cap B_{\varepsilon}\left(x_{0}\right)$. If $F$ is never zero, we deduce that this function is in $B V_{l o c}$, thus proving that $\{\psi=$ $\left.\psi\left(x_{0}\right)\right\} \cap B_{\varepsilon}\left(x_{0}\right)$ and $\operatorname{supp}(\mu) \cap B_{\varepsilon}\left(x_{0}\right)$ have finite perimeter. Here, we use (3.9) and, more generally, the fact that if $E$ is a subset of $G$ and $|G \backslash E|=0$, then $E$ and $G$ have the same perimeter. 


\section{More Singular than Newtonian Repulsion}

In this section, we consider kernel that are more repulsive than Newtonian. We remind the reader that we assume that $W$ satisfies $(\mathrm{H} 1)$ and $\left(\mathrm{H} 2^{s}\right)$. Moreover, we consider a $\varepsilon$-local minimizer $\mu$ (in the sense of Definition 2.1) such that $\operatorname{supp}(\mu)$ is compact. We thus have $\operatorname{supp}(\mu) \subset B_{R}(0)$ for some large $R$. As discussed in Sect. 3, we can cut off the values of $W_{a}(x)$ outside a large ball since those values have no influence on the values of the potential $\psi$ in $B_{R}$, or on the energy $E[\mu]$. Throughout this section, we can therefore assume

$\left(\mathrm{H}^{S}\right)$ The kernel $W_{a}$ is compactly supported, and there exists a constant $M$ such that

$$
\left\|(-\Delta)^{s} W_{a}\right\|_{L^{p}\left(B_{1}(x)\right)} \leq M \quad \text { for all } x \in \mathbb{R}^{N}
$$

where $p \in\left(\frac{N}{2 s}, \infty\right]$.

6.1. Continuity of $\psi$ : Proof of Proposition 3.8. A key tool in the proof of the continuity of $\psi$ in the Newtonian case was the mean-value formula for Laplace's equation. Fortunately, this formula can be generalized to the fractional Laplace equation (see [51]). However, as usual with fractional powers of the Laplacian, this formula is non local which complicates the proof of Proposition 3.8. For that reason, even though the main ideas are the same as in the proof of Proposition 3.2, we present here the proof of Proposition 3.8 in full details.

Our first task is to state this generalized mean-value formula. For that, we recall that $V_{s}(x)=\frac{c_{N, s}}{|x|^{N-2 s}}$ is the fundamental solution of $(-\Delta)^{s}$. Proceeding as in [51], we define the function $\Gamma$ (we drop the index $s$ for the sake of simplicity) as follows:

$$
\Gamma(x)= \begin{cases}V_{s}(s) & \text { in }|x| \geq 1 \\ A x^{2}+B & \text { in }|x| \leq 1\end{cases}
$$

where the constant $A$ and $B$ are chosen in such a way that $\Gamma$ be a $C^{1,1}$ function. Given $\lambda>0$, we then scale $\Gamma$ in the following way:

$$
\Gamma_{\lambda}(x)=\frac{1}{\lambda^{N-2 s}} \Gamma\left(\frac{x}{\lambda}\right) .
$$

Note that the function $\Gamma_{\lambda}(x)$ coincides with $V_{s}(x)$ outside of the ball of radius $\lambda$. Furthermore, if $\lambda_{1} \leq \lambda_{2}$, then $\Gamma_{\lambda_{1}} \geq \Gamma_{\lambda_{2}}$. Finally, we define

$$
\gamma_{\lambda}:=(-\Delta)^{s} \Gamma_{\lambda} .
$$

Note that $\gamma_{\lambda}(x)=\frac{1}{\lambda^{N}} \gamma_{1}(x / \lambda)$. We then have the following result.

Lemma 6.1 [51].

(i) For all $\lambda>0, \gamma_{\lambda}$ is a positive integrable continuous function of unit mass.

(ii) $x \mapsto \gamma_{\lambda}(x)$ decays like $\frac{1}{|x|^{N+2 s}}$ as $|x| \rightarrow \infty$.

(iii) The family of function $\left\{\gamma_{\lambda}\right\}_{\lambda>0}$ is an approximation of the identity.

We can now state the mean-value formula for the fractional Laplace equation:

Lemma 6.2. Assume that $u$ is an upper semi-continuous, compactly supported function and $\Omega$ an open set in $\mathbb{R}^{N}$. 
(i) If $(-\Delta)^{s} u \geq 0$ in $\Omega$, then for all $x \in \Omega$ and for all $0<\lambda<d(x, \partial \Omega)$, it holds that

$$
u(x) \geq u * \gamma_{\lambda}(x) .
$$

(ii) If $(-\Delta)^{s} u \in L_{l o c}^{p}\left(\mathbb{R}^{N}\right)$ for some $p>\frac{N}{2 s}$, then for all $x \in \mathbb{R}^{N}$ and for all $\lambda \in(0,1)$, it holds that

$$
u(x) \geq u * \gamma_{\lambda}(x)-C\left\|(-\Delta)^{s} u\right\|_{L^{p}\left(B_{1}(x)\right)} \lambda^{\alpha},
$$

with $\alpha=2 s-\frac{N}{p}$, where $C$ is a constant depending only on $N, s$ and $p$.

We recall the proof of this lemma in Appendix B (for the sake of completeness). Note that the assumption that $u$ be compactly supported is clearly not necessary for such a formula to hold, but $u$ must satisfy some appropriate integrability condition. For our purpose, it is enough to consider this simpler case. We can now prove the continuity of the potential function.

Lemma 6.3. Let $\mu \in \mathcal{P}\left(\mathbb{R}^{N}\right)$ compactly supported, and assume that $(\mathrm{H} 1),\left(\mathrm{H} 2^{s}\right)$ and $\left(\mathrm{H} 3^{s}\right)$ hold. Then there exists a constant $C$ depending on $N, s, p$ and the constant $M$ appearing in (6.1) such that the potential function $\psi=W * \mu$ satisfies

$$
\psi(x) \geq \psi * \gamma_{\lambda}(x)-C \lambda^{\alpha}
$$

for all $x \in \mathbb{R}^{N}$ and for all $\lambda \in(0,1)$ and with $\alpha=2 s-\frac{N}{p}$.

Furthermore,

$$
\psi(x) \leq \psi * \gamma_{\lambda}(x)+C_{*} \lambda^{2 s}
$$

for all $x \in \mathbb{R}^{N} \backslash \operatorname{supp}(\mu)$ and for all $0<\lambda<d(x, \operatorname{supp}(\mu))$, where $C_{*}$ is the constant appearing in (3.11).

Proof. We can always assume that $\psi(x)<\infty$ (since otherwise (6.2) clearly holds). We have $W=V_{s}+W_{a}$, where $V_{s}$ satisfies the conditions of Lemma 6.2(i) and $W_{a}$ satisfies the conditions of Lemma 6.2(ii), so we can write

$$
W(x-y) \geq W * \gamma_{\lambda}(x-y)-C\left\|(-\Delta)^{s} W_{a}\right\|_{L^{p}\left(B_{1}(x-y)\right)} \lambda^{\alpha}
$$

for all $x, y \in \mathbb{R}^{N} \times \mathbb{R}^{N}$ and for all $\lambda \in(0,1)$ where $C$ only depends on $N, s$ and $p$. Using (6.1), we deduce

$$
\psi(x)=\int_{\mathbb{R}^{N}} W(x-y) d \mu(y) \geq \int_{\mathbb{R}^{N}} W * \gamma_{\lambda}(x-y) d \mu(y)-C(N, s, p, M) \lambda^{\alpha}
$$

and Fubini-Tonelli theorem implies

$$
\int_{\mathbb{R}^{N}} W * \gamma_{\lambda}(x-y) d \mu(y)=\psi * \gamma_{\lambda}(x) .
$$

Note that the integral in the left hand side is finite due to the decay at infinity of $V_{s}$. The first part of the result follows.

To prove the second part of the lemma, we fix $x \in \mathbb{R}^{N} \backslash \operatorname{supp}(\mu)$ and $0<\lambda<$ $d(x, \operatorname{supp}(\mu))$ and we consider $B=B_{r_{0}}(x)$ the biggest ball around $x$ which is contained in $\mathbb{R}^{N} \backslash \operatorname{supp}(\mu)$ (so $r_{0}=d(x, \operatorname{supp}(\mu))$ ). We then define $v=C_{*} V_{s} * \chi_{B}$, which 
satisfies $(-\Delta)^{s} v=C_{*} \chi_{B}$. Proceeding as in the proof of the mean value formula (see Appendix B), we can write (integration by parts):

$$
\int_{\mathbb{R}^{N}}(-\Delta)^{s} v(x+y)\left(\Gamma_{\lambda_{1}}(y)-\Gamma_{\lambda}(y)\right) d y=v * \gamma_{\lambda_{1}}(x)-v * \gamma_{\lambda}(x)
$$

and so

$$
\int_{\mathbb{R}^{N}} C_{*} \chi_{B}(x+y)\left(\Gamma_{\lambda_{1}}(y)-\Gamma_{\lambda}(y)\right) d y=v * \gamma_{\lambda_{1}}(x)-v * \gamma_{\lambda}(x)
$$

Letting $\lambda_{1}$ go to zero, we deduce

$$
C_{*} \int_{B_{r_{0}}(0)}\left(V_{s}(y)-\Gamma_{\lambda}(y)\right) d y=v(x)-v * \gamma_{\lambda}(x)
$$

Finally, the scaling of the integral in the left hand side (recall that $\Gamma_{\lambda}(x)=V_{S}(x)$ for $|x| \geq \lambda$ ) gives

$$
v(x)-v * \gamma_{\lambda}(x)=C_{*} C \lambda^{2 s}
$$

Hypothesis $\left(\mathrm{H}^{s}\right)$ implies in particular that $(-\Delta)^{s} \psi \leq C_{*}$ in $\mathbb{R}^{N} \backslash \operatorname{supp}(\mu)$ and so

$$
(-\Delta)^{s}(\psi-v) \leq 0 \text { in } B .
$$

Using Lemma 6.2 (with $u=-(\psi-v)$ ), we get that $\psi(x)-v(x) \leq \psi * \gamma_{\lambda}(x)-v * \gamma_{\lambda}(x)$. Hence,

$$
\psi(x) \leq \psi * \gamma_{\lambda}(x)+\left(v(x)-v * \gamma_{\lambda}(x)\right)=\psi * \gamma_{\lambda}(x)+C_{*} C \lambda^{2 s}
$$

which is exactly (6.3).

Lemma 6.4. Assume that $W$ satisfies $(\mathrm{H} 1),\left(\mathrm{H} 2^{s}\right)$, and $\left(\mathrm{H}^{s}\right)^{2}$. Then the potential function $\psi=W * \mu$ is bounded below in $\mathbb{R}^{N}$.

Proof. Clearly, we have $\psi-W_{a} * \mu=V_{s} * \mu \geq 0$, so $\psi \geq W_{a} * \mu$. Furthermore $\left(\mathrm{H} 2^{s}\right)$ and $\left(\mathrm{H} 3^{s}\right)$ implies that $W_{a}$ is continuous and compactly supported, and thus bounded. The result follows.

Using the previous lemmas we can now prove the following consequences.

Corollary 6.5. Assume that $W$ satisfies $(\mathrm{H} 1),\left(\mathrm{H} 2^{s}\right)$, and $\left(\mathrm{H}^{s}\right)$. Let $\mu$ be a $\varepsilon$-local minimizer in the sense of Definition 2.1. Then any point $x_{0} \in \operatorname{supp}(\mu)$ is a local minimizer of $\psi=W * \mu$ in the sense that

$$
\psi\left(x_{0}\right) \leq \psi(x) \quad \text { for all } x \in B_{\varepsilon}\left(x_{0}\right) .
$$

Furthermore, we have

$$
\psi(x)=\psi\left(x_{0}\right) \quad \text { for all } x \in \operatorname{supp}(\mu) \cap B_{\varepsilon}\left(x_{0}\right) .
$$


Proof. First, we note that (6.2) implies

$$
\psi(x) \geq \lim _{\lambda \rightarrow 0} \psi * \gamma_{\lambda}(x) \text { for all } x .
$$

Next, we recall that $\psi(x) \geq \psi\left(x_{0}\right)$ for a.e. $x \in B_{\epsilon}\left(x_{0}\right)$. Using Lemmas 6.4 and 6.3, we can thus write, for $x \in B_{\varepsilon}\left(x_{0}\right)$ :

$$
\begin{aligned}
\psi * \gamma_{\lambda}(x) & =\int_{B_{\bar{\varepsilon}}(0)} \psi(x-y) \gamma_{\lambda}(y) d y+\int_{\mathbb{R}^{N} / B_{\bar{\varepsilon}}(0)} \psi(x-y) \gamma_{\lambda}(y) d y \\
& \geq \psi\left(x_{0}\right) A_{1}(\bar{\varepsilon}, \lambda)+\left(\inf _{\mathbb{R}^{N}} \psi\right) A_{2}(\bar{\varepsilon}, \lambda)
\end{aligned}
$$

for some $\bar{\varepsilon}<\varepsilon-\left|x-x_{0}\right|$ where

$$
A_{1}(\bar{\varepsilon}, \lambda)=\int_{B_{\bar{\varepsilon}}(0)} \gamma_{\lambda}(y) d y \quad \text { and } \quad A_{2}(\bar{\varepsilon}, \lambda)=\int_{\mathbb{R}^{N} / B_{\bar{\varepsilon}}(0)} \gamma_{\lambda}(y) d y .
$$

Lemma 6.1 implies

$$
\lim _{\lambda \rightarrow 0} A_{1}(\bar{\varepsilon}, \lambda)=1, \quad \lim _{\lambda \rightarrow 0} A_{2}(\bar{\varepsilon}, \lambda)=0,
$$

which gives (6.4). The equality for $x \in \operatorname{supp}(\mu)$ follows from Remark 2.3.

We can finally prove the first main result of this section: The continuity of the potential $\psi$. As for the Newtonian case, the proof follows essentially Evans proof for the continuity of the solution of the obstacle problem, which was adapted to the fractional case by Silvestre [51].

Proof of Proposition 3.8. First, we consider a sequence $x_{k} \in \mathbb{R}^{N}$ such that $x_{k} \rightarrow x_{0} \notin$ $\operatorname{supp}(\mu)$. By definition of $\operatorname{supp}(\mu)$, there exists a ball $B_{r}\left(x_{0}\right)$ such that $\mu\left(B_{r}\left(x_{0}\right)\right)=0$. We can thus write

$$
\psi(x)=\int_{|x-y| \geq r / 2} W(x-y) d \mu(y) \text { for all } x \in B_{r / 2}\left(x_{0}\right)
$$

and we can always assume that $x_{k} \in B_{r / 2}\left(x_{0}\right)$ (for $k$ large enough). Hypothesis $\left(\mathrm{H} 2^{s}\right.$ ) implies that $W(x)$ is continuous in $\left\{x \in \mathbb{R}^{N} ;|x| \geq r / 2\right\}$ and due to $\left(\mathrm{H}^{3}\right) W$ is compactly supported, so

$$
\lim _{k \rightarrow \infty} \psi\left(x_{k}\right)=\psi\left(x_{0}\right) .
$$

This proves the continuity of $\psi$ outside of $\operatorname{supp}(\mu)$.

We now fix $x_{0} \in \operatorname{supp}(\mu)$ and consider a sequence $x_{k} \in \mathbb{R}^{N}$ such that $x_{k} \rightarrow x_{0}$ as $k \rightarrow \infty$. We can always assume that $x_{k} \in B_{\varepsilon}\left(x_{0}\right)$ for all $k$. In particular, if $x_{k} \in \operatorname{supp}(\mu)$ then (6.5) implies $\psi\left(x_{k}\right)=\psi\left(x_{0}\right)$, and so once again $\lim _{k \rightarrow \infty} \psi\left(x_{k}\right)=\psi\left(x_{0}\right)$. So as in the Newtonian case, we are reduced to considering a subsequence (still denoted $x_{k}$ ) such that

$$
x_{k} \notin \operatorname{supp}(\mu) \text { for all } k, \quad \lim _{k \rightarrow \infty} x_{k}=x_{0} \in \operatorname{supp}(\mu) .
$$

Since $x_{k} \in B_{\varepsilon}\left(x_{0}\right)$ for all $k,(6.4)$ implies

$$
\psi\left(x_{k}\right) \geq \psi\left(x_{0}\right) \quad \text { for all } k .
$$


For all $k$ let $y_{k}$ be the closest point to $x_{k}$ which is in $\operatorname{supp}(\mu) \cap \overline{B_{\varepsilon / 2}}\left(x_{0}\right)$. Again, for $k$ large enough, we can assume that $y_{k} \in B_{\varepsilon}\left(x_{0}\right)$ and so (6.5) implies that $\psi\left(y_{k}\right)=\psi\left(x_{0}\right)$. We denote $\delta_{k}=\left|x_{k}-y_{k}\right|$. Note that $\delta_{k}$ is the distance of $x_{k}$ to $\operatorname{supp}(\mu)$ for $k$ large enough, and so $\delta_{k} \leq\left|x_{k}-x_{0}\right| \rightarrow 0$. Inequality (6.2) implies

$$
\psi\left(x_{0}\right)=\psi\left(y_{k}\right) \geq \psi * \gamma_{\delta_{k}}\left(y_{k}\right)-C \delta_{k}^{\alpha} .
$$

Let us define now

$$
C_{0}=\inf _{x \in \mathbb{R}} \frac{\gamma_{1}(x+e)}{\gamma_{1}(x)},
$$

where $e$ is any unit vector. The infimum is achieved and it is positive, because $\lim _{|x| \rightarrow \infty} \frac{\gamma_{1}(x+e)}{\gamma_{1}(x)}=1$. Furthermore, by symmetry of $\gamma$ it does not depend on the choice of $e$. Setting $e=\frac{x_{k}-y_{k}}{\delta_{k}}$, we obtain

$$
\begin{aligned}
\gamma_{\delta_{k}}\left(x-y_{k}\right)-C_{0} \gamma_{\delta_{k}}\left(x-x_{k}\right) & =\frac{1}{\delta_{k}^{N}}\left(\gamma_{1}\left(\frac{x-x_{k}}{\delta_{k}}+\frac{x_{k}-y_{k}}{\delta_{k}}\right)-C_{0} \gamma_{1}\left(\frac{x-x_{k}}{\delta_{k}}\right)\right) \\
& =\frac{1}{\delta_{k}^{N}}\left(\gamma_{1}\left(\frac{x-x_{k}}{\delta_{k}}+e\right)-C_{0} \gamma_{1}\left(\frac{x-x_{k}}{\delta_{k}}\right)\right) \\
& \geq 0 .
\end{aligned}
$$

We now use (6.2) and (6.3) to write, for $k$ large enough,

$$
\begin{aligned}
\psi\left(x_{0}\right)=\psi\left(y_{k}\right) & \geq \psi * \gamma_{\delta_{k}}\left(y_{k}\right)-C \delta_{k}^{\alpha} \\
& =C_{0} \psi * \gamma_{\delta_{k}}\left(x_{k}\right)+\int_{\mathbb{R}^{N}}\left(\gamma_{\delta_{k}}\left(y-y_{k}\right)-C_{0} \gamma_{\delta_{k}}\left(y-x_{k}\right)\right) \psi(y) d y-C \delta_{k}^{\alpha} \\
& \geq C_{0} \psi\left(x_{k}\right)-C_{0} C \delta_{k}^{2 s}+I_{1}+I_{2}-C \delta_{k}^{\alpha},
\end{aligned}
$$

where

$$
I_{1}=\int_{B_{\sqrt{\delta_{k}}}\left(y_{k}\right)}\left(\gamma_{\delta_{k}}\left(y-y_{k}\right)-C_{0} \gamma_{\delta_{k}}\left(y-x_{k}\right)\right) \psi(y) d y
$$

and

$$
I_{2}=\int_{\mathbb{R}^{N} \backslash B \sqrt{\delta_{k}}\left(y_{k}\right)}\left(\gamma_{\delta_{k}}\left(y-y_{k}\right)-C_{0} \gamma_{\delta_{k}}\left(y-x_{k}\right)\right) \psi(y) d y
$$

Using (6.7) and the fact that $\psi(y) \geq \psi\left(x_{0}\right)$ in $B_{\sqrt{\delta_{k}}}\left(y_{k}\right)$, for $k$ large enough, we get

$$
\begin{aligned}
I_{1} & \geq \psi\left(x_{0}\right) \int_{B_{\sqrt{\delta_{k}}}\left(y_{k}\right)}\left[\gamma_{\delta_{k}}\left(y-y_{k}\right)-C_{0} \gamma_{\delta_{k}}\left(y-x_{k}\right)\right] d y \\
& \geq \psi\left(x_{0}\right)\left[1-C_{0}-\varepsilon_{k}\right]
\end{aligned}
$$

where

$$
\varepsilon_{k}=\int_{\mathbb{R}^{N} \backslash B \sqrt{\delta_{k}}\left(y_{k}\right)}\left[\gamma_{\delta_{k}}\left(y-y_{k}\right)-C_{0} \gamma_{\delta_{k}}\left(y-x_{k}\right)\right] d y
$$


here we used the fact that $\gamma_{\lambda}$ has unit mass for all $\lambda$. Making the change of variable $z=\frac{y-y_{k}}{\delta_{k}}$ and using the notation $e=\frac{y_{k}-x_{k}}{\delta_{k}}$, we find

$$
\varepsilon_{k}=\int_{\mathbb{R}^{N} \backslash B \sqrt{\delta_{k}}(0)}\left(\gamma_{1}(z)-C_{0} \gamma_{1}(z+e)\right) d z
$$

so

$$
\lim _{k \rightarrow \infty} \varepsilon_{k}=0
$$

Now we turn to $I_{2}$. Using (6.7) and Lemma 6.4, we get

$$
I_{2} \geq\left(\inf _{\mathbb{R}^{N}} \psi\right) \int_{\mathbb{R}^{N} \backslash B \sqrt{\delta_{k}}}\left(y_{k}\right)\left(\gamma_{\delta_{k}}\left(y-y_{k}\right)-C_{0} \gamma_{\delta_{k}}\left(y-x_{k}\right)\right) d y=\varepsilon_{k} \inf _{\mathbb{R}^{N}} \psi
$$

Combining all the above estimates, we conclude that, for $k$ large enough,

$$
\begin{aligned}
\psi\left(x_{0}\right)= & \psi\left(y_{k}\right) \geq C_{0} \psi\left(x_{k}\right)+\left(1-C_{0}\right) \psi\left(x_{0}\right)-C \delta_{k}^{\alpha}-C_{0} C_{*} \delta_{k}^{2 s} \\
& -\varepsilon_{k}\left(\psi\left(x_{0}\right)+\inf _{\mathbb{R}^{N}} \psi\right) .
\end{aligned}
$$

We deduce

$$
\limsup _{k \rightarrow \infty} \psi\left(x_{k}\right) \leq \psi\left(x_{0}\right)
$$

which, together with (6.6), implies

$$
\lim _{k \rightarrow \infty} \psi\left(x_{k}\right)=\psi\left(x_{0}\right)
$$

and completes the proof.

6.2. Regularity of the density function. In order to apply known regularity results for the fractional obstacle problem (as found, for instance, in [51]), we need to show that $\psi$ solves a fractional obstacle problem in the whole of $\mathbb{R}^{N}$.

It is possible to do this as follows: The set $\operatorname{supp}(\mu)+B_{\varepsilon / 4}=\{x+y ; x \in \operatorname{supp}(\mu), y \in$ $\left.B_{\varepsilon / 4}(0)\right\}$ is an open set in $B_{R+1}(0)$. In particular, it is the countable union of its connected components $A_{i}$. Furthermore, since $\operatorname{supp}(\mu)$ is compact, there are only finitely many $A_{i}$.

For all $i$, any two points $x_{1}, x_{2}$ in $\operatorname{supp}(\mu) \cap A_{i}$ will satisfy $\psi\left(x_{1}\right)=\psi\left(x_{2}\right)$, by the minimality of the connected component and Corollary 6.5. We define $D_{i}=\operatorname{supp}(\mu) \cap A_{i}$, we denote $C_{i}=\psi||_{D_{i}}$ and we consider a smooth function $f$ such that

$$
\begin{aligned}
& f \leq C_{i} \text { in } A_{i} \\
& f=C_{i} \text { on } D_{i}+B_{\frac{\varepsilon}{16}} \\
& f=\inf W \text { outside } \cup_{i} D_{i}+B_{\varepsilon / 8} .
\end{aligned}
$$


We can find such smooth function, because $D_{i}$ are at least separated $\varepsilon / 4$ from each other, and if they are closer than $\varepsilon$ then the constant $C_{i}$ has to match because of Corollary 6.5. The potential function $\psi$ then solves the following obstacle problem in $\mathbb{R}^{N}$ :

$$
\begin{cases}\psi \geq f, \quad-\Delta \psi \geq-F(x) & \text { in } \mathbb{R}^{N} \\ -(\Delta)^{s} \psi=-F(x), & \text { in }\{\varphi>f\}\end{cases}
$$

where $F=-(-\Delta)^{s} W_{a} * \mu$.

Using this obstacle problem formulation, we can use the following proposition which is the fractional analog of Proposition 5.2 (See Silvestre [51]):

Proposition 6.6. Let $\psi$ be the solution of the obstacle problem (6.8). If $f \in C^{2}\left(\mathbb{R}^{N}\right)$ and $W_{a} * \mu$ is in $C^{\beta}\left(\mathbb{R}^{N}\right)$ with $\beta>0$. Then $\psi \in C^{\alpha}\left(\mathbb{R}^{N}\right)$ for every $\alpha<\min (\beta, 1+s)$ (with the notation $C^{\alpha}=C^{1, \alpha-1}$ if $\alpha>1$ ).

Proof of Theorem 3.10. We can now prove our main result by proceeding as in the proof of Theorem 3.4 (ii):

First, $\left(\mathrm{H} 2^{s}\right)$ implies that $(-\Delta)^{s} W_{a} * \mu \in L^{p}$ with $p>\frac{N}{2 s}$ and so $W_{a} * \mu \in C^{\alpha}\left(\mathbb{R}^{N}\right)$ for $\alpha=2 s-\frac{N}{p}$. Proposition 6.6 implies that $\psi \in C^{\alpha}$. Since $\mu=(-\Delta)^{s} \psi-(-\Delta)^{s} W_{a} * \mu$, we can write

$$
W_{a} * \mu=W_{a} *(-\Delta)^{s} \psi-W_{a} *\left((-\Delta)^{s} W_{a} * \mu\right)=(-\Delta)^{s} W_{a} *\left(\psi+W_{a} * \mu\right) .
$$

Using the fact that $(-\Delta)^{s} W_{a} \in W^{\varepsilon, 1}\left(\mathbb{R}^{N}\right)$ and Lemma 5.1, this implies that

$$
W_{a} * \mu \in \mathcal{C}^{\alpha+\varepsilon}\left(\mathbb{R}^{N}\right) .
$$

We iterate this argument until we get that $W_{a} * \mu \in C^{1, s}\left(\mathbb{R}^{N}\right)$, which then implies (by Proposition 6.6) that $\psi \in C^{1, \gamma}\left(\mathbb{R}^{N}\right)$ for all $\gamma<s$.

Finally, this implies that $\mu=(-\Delta)^{s} \psi-(-\Delta)^{s} W_{a} * \mu \in C^{\beta}\left(\mathbb{R}^{N}\right)$ for all $\beta<1-s$ (see Proposition 2.7 in [51]) and complete the proof of Theorem 3.10.

\section{Uniqueness: Proof of Theorem 3.12}

We now turn to the proof of our uniqueness result when $W_{a}=K|x|^{2}$. We assume that $\mu_{0} \in \mathcal{P}\left(\mathbb{R}^{N}\right)$ is $d_{2}$ local minimizer of $E$, and we recall (see comment before Theorem 3.12) that such a minimizer is compactly supported. We will only prove the result for potential satisfying $(\mathrm{H} 2 \mathrm{~s})$, since the Newtonian case is easier.

From our work in the previous section, we know that $\mu_{0}=\rho_{0} d \mathcal{L}^{N}$ where $\rho_{0}$ is a continuous function. Furthermore, we recall that for $d_{2}$-minimizers, the constant $C_{i}$ is the same on all connected component of $\operatorname{supp}\left(\mu_{0}\right)$ (and equal to $2 E\left[\mu_{0}\right]$ ). We deduce that $h=V_{s} * \mu_{0}$ satisfies

$$
\left\{\begin{aligned}
h & \geq C-K|x|^{2} * \rho_{0}, & & \text { in } \mathbb{R}^{N} \\
(-\Delta)^{s} h & \geq 0, & & \text { in } \mathbb{R}^{N} \\
(-\Delta)^{s} h & =0, & & \text { in }\left\{h>C-K|x|^{2} * \rho_{0}\right\}
\end{aligned}\right.
$$

for some constant $C$.

By translational invariance, we can always assume that the center of mass of $\mu_{0}$ is zero, and so

$$
K|x|^{2} * \rho_{0}=K\left[|x|^{2}+\int_{\mathbb{R}^{N}}|y|^{2} \rho_{0}(y) d y\right]=K|x|^{2}+C_{\rho_{0}} .
$$


Finally, using the fact that $\rho_{0} \in L^{\infty}\left(\mathbb{R}^{N}\right)$ and that $\rho_{0}$ has compact support, it is easy to see that $h(x) \rightarrow 0$ as $|x| \rightarrow \infty$. Hence, $h$ solves the following fractional obstacle problem in $\mathbb{R}^{N}$ :

$$
\left\{\begin{array}{rlrl}
\varphi & \geq C-K|x|^{2} * \rho_{0}, & & \text { in } \mathbb{R}^{N} \\
(-\Delta)^{S} \varphi & \geq 0, & & \text { in } \mathbb{R}^{N} \\
(-\Delta)^{S} \varphi & =0, & & \text { in }\left\{\varphi>C-K|x|^{2} * \rho_{0}\right\} \\
\varphi & \rightarrow 0, & \text { when } x \rightarrow \infty,
\end{array}\right.
$$

for some constant $C>0$.

By Theorem 3.1 in [16], we know that for any given $C>0(7.1)$ has a unique solution $\varphi_{C}$. Furthermore, this solution is radially symmetric and satisfies the scaling property:

$$
\varphi_{C}(x)=C \varphi_{1}\left(\frac{x}{C^{\frac{1}{2}}}\right) .
$$

In particular, the function

$$
\rho_{C}(x):=(-\Delta)^{s} \varphi_{C}(x)=C^{1-s} \rho_{1}\left(\frac{x}{C^{\frac{1}{2}}}\right)
$$

satisfies

$$
\int_{\mathbb{R}^{n}} \rho_{C}(x) d x=C^{\frac{n}{2}+1-s} \int_{\mathbb{R}^{n}} \rho_{1}(x) d x .
$$

Therefore, there is a unique $C_{0}>0$ for which $\rho_{C_{0}}$ has unit mass. It follows that $\rho_{0}=\rho_{C_{0}}$, which implies the uniqueness of $\rho_{0}$ and the fact that it is radially symmetric.

In the Newtonian case, one can also show that the coincidence set is a ball, by using the maximum principle. The case involving the fractional Laplacian is harder to characterize due to the non-locality of the problem.

7.1. A different proof in the Newtonian case. In this section, we give an alternative proof of the uniqueness of global minimizer in the Newtonian case. To simplify the notations, we assume that $W=V+\frac{|x|^{2}}{2 N}$.

In particular, we have $F(x)=1$ and so minimizers of our energy (global or local) are of the form $\mu_{0}=\chi_{\Omega} d \mathcal{L}^{N}$ with $|\Omega|=1$. Using translation invariance, we can further assume that $\mu_{0}$ has zero center of mass.

Next, we use a simple scaling argument to derive an important relation: Consider the dilation maps $\mathcal{T}_{\lambda}: \mathbb{R}^{N} \rightarrow \mathbb{R}^{N}$ defined by $\mathcal{T}_{\lambda}(x)=\lambda x$ with $\lambda>0$. Given any $\mu \in \mathcal{P}\left(\mathbb{R}^{N}\right)$, we define $\mu^{\lambda}=\mathcal{T}_{\lambda} \# \mu$. Using the fact that the (Newtonian) repulsive and (quadratic) attractive parts of the potential $W$ scale differently, we can re-write the energy of $\mu^{\lambda}$ in the following way:

$$
E\left[\mu^{\lambda}\right]=\frac{1}{\lambda^{N-2}} E_{r}[\mu]+\lambda^{2} E_{a}[\mu]
$$

where $E_{r}[\mu]$ is the energy associated to the repulsive Newtonian interaction potential $V$ and $E_{a}[\mu]$ the energy associated to the attractive quadratic confinement $|x|^{2} / 2 N$. 
For any $d_{2}$-local minimizer, the function $\lambda \mapsto E\left[\mu_{0}^{\lambda}\right]$ must have a minimum at $\lambda=1$. By differentiating (7.2) we deduce

$$
\left.\frac{d E\left[\mu_{0}^{\lambda}\right]}{d \lambda}\right|_{\lambda=1}=(2-N) E_{r}\left[\mu_{0}\right]+2 E_{a}\left[\mu_{0}\right]=0 .
$$

Using this identity, we can rewrite the energy for $\mu_{0}$ as

$$
E\left[\mu_{0}\right]=\left(\frac{N-2}{2}+1\right) E_{a}\left[\mu_{0}\right]=\left(\frac{N-2}{2}+1\right) \int_{\mathbb{R}^{N}} \frac{|x|^{2}}{N} d \mu_{0}(x) .
$$

It remains to see that this implies that $\mu_{0}=\chi_{B_{m}}$ where $B_{m}$ is the ball centered at 0 with $\left|B_{m}\right|=1$. This follows from the following two facts:

1. Among all sets $\Omega$ of unit area and zero center of mass, the unique minimizer of

$$
\int_{\Omega}|x|^{2} d x
$$

is the ball $B_{m}$.

2. The measure $\mu_{0}=\chi_{B_{m}}$ satisfies (7.3).

The second point is proved in [27], and it follows from the fact that $\chi_{B_{m}}$ is a $d_{2}$-local minimizer of $E$ under dilation.

In order to prove the first point, we consider any measurable set $\Omega$ with zero center of mass and measure 1 , and we decompose it as $\Omega=\Omega_{a} \cup \Omega_{b}$ with $\Omega_{a}=\Omega \cap B_{m}$ and $\Omega_{b}=\Omega / \Omega_{a}$. Take the set $B_{a}=B_{m} / \Omega_{a}$. Since $\left|B_{m}\right|=|\Omega|=1$, we must have $\left|\Omega_{b}\right|=\left|B_{a}\right|$. If $\left|B_{a}\right|=\left|\Omega_{b}\right|>0$, then we have

$$
\begin{aligned}
\int_{B_{m}}|x|^{2} d x & =\int_{\Omega_{a}}|x|^{2} d x+\int_{B_{a}}|x|^{2} d x \\
& <\int_{\Omega_{a}}|x|^{2} d x+r_{m}^{2}\left|B_{a}\right|=\int_{\Omega_{a}}|x|^{2} d x+r_{m}^{2}\left|\Omega_{b}\right| \\
& <\int_{\Omega_{a}}|x|^{2} d x+\int_{\Omega_{b}}|x|^{2} d x=\int_{\Omega}|x|^{2} d x
\end{aligned}
$$

where $r_{m}$ denotes the radius of $B_{m}$. This completes the proof of uniqueness of global minimizers.

Note that if $\mu_{0}$ is a $d_{2}$-local minimizer (rather than a global minimizer) with zero center of mass, we still have that $\mu_{0}=\chi_{\Omega} d \mathcal{L}^{N}$ with $|\Omega|=1$, and (7.3) still holds. Furthermore, if $\Omega$ is not the ball $B_{m}$, we can show that by moving a small amount of mass to a small ball in $B_{m} \backslash \Omega$, we obtain a set $\Omega^{\prime}$ such that $\chi_{\Omega^{\prime}}$ is close to $\chi_{\Omega}$ in the $d_{2}$ topology and

$$
\int_{\Omega^{\prime}}|x|^{2} d x<\int_{\Omega}|x|^{2} d x
$$

However, since $\chi_{\Omega^{\prime}}$ might not satisfy (7.3), so we cannot derive a contradiction. Therefore, this proof only applies to global minimizer, and we note that the fact that the measure $\mu_{0}=\chi_{B_{m}}$ satisfies (7.3) is really essential. 
Acknowledgements. JAC acknowledges support from projects MTM2011-27739-C04-02, 2009-SGR-345 from Agència de Gestió d'Ajuts Universitaris i de Recerca-Generalitat de Catalunya, the Royal Society through a Wolfson Research Merit Award, and the Engineering and Physical Sciences Research Council (UK) Grant Number EP/K008404/1. AM was partially supported by NSF Grant DMS-1201426. The authors thank MSRI at Berkeley where part of this work was carried over during the program on Optimal Transport.

Open Access This article is distributed under the terms of the Creative Commons Attribution 4.0 International License (http://creativecommons.org/licenses/by/4.0/), which permits unrestricted use, distribution, and reproduction in any medium, provided you give appropriate credit to the original author(s) and the source, provide a link to the Creative Commons license, and indicate if changes were made.

\section{A. Mean Value Formula}

For the sake of completeness, we recall here the derivation of the mean-value formula (Lemma 4.1).

We recall that $V(x)$ denotes the fundamental solution of the Laplace equation, and we remove the singularity at $x=0$ by gluing a paraboloid to $V$ in the ball of radius 1 . More precisely, we define a $C^{1,1}\left(\mathbb{R}^{N}\right)$ function $\Gamma$ by

$$
\begin{cases}\Gamma(x)=V(x) & \text { in }|x|>1 \\ \Gamma(x)=-\frac{1}{2\left|B_{1}\right|}|x|^{2}+C & \text { in }|x| \leq 1\end{cases}
$$

Given $\lambda>0$, also introduce $\Gamma_{\lambda}=\frac{1}{\lambda^{N-2}} \Gamma\left(\frac{x}{\lambda}\right)$. We note that the function $\Gamma_{\lambda}$ coincides with $V(x)$ outside of the ball of radius $\lambda$. Furthermore, if $\lambda_{1} \leq \lambda_{2}$, then $\Gamma_{\lambda_{1}} \geq \Gamma_{\lambda_{2}}$. Finally, we note that $-\Delta \Gamma_{\lambda}$ is an approximation of the identity. In fact, we have $-\Delta \Gamma_{\lambda}=\frac{1}{\left|B_{\lambda}\right|} \chi_{B_{\lambda}}$. Using these facts, we can now prove Lemma 4.1:

Proof of Lemma 4.1. Take $x \in B_{R}$ and $0<\lambda_{1}<\lambda_{2}<1$, then $\Gamma_{\lambda_{1}}-\Gamma_{\lambda_{2}}$ is $C^{1,1}\left(\mathbb{R}^{N}\right)$ and compactly supported on the ball of radius $\lambda_{2}$. Using Hölder inequality we get

$$
\int_{\mathbb{R}^{N}} \Delta u(x+y)\left(\Gamma_{\lambda_{1}}(y)-\Gamma_{\lambda_{2}}(y)\right) d y \leq\|\Delta u\|_{L^{p}\left(B_{1}(x)\right)}\left\|\Gamma_{\lambda_{1}}-\Gamma_{\lambda_{2}}\right\|_{L^{q}\left(B_{\lambda_{2}}\right)} .
$$

Integrating by parts the left hand side also gives

$$
\int_{\mathbb{R}^{N}} \Delta u(x+y)\left(\Gamma_{\lambda_{1}}(y)-\Gamma_{\lambda_{2}}(y)\right) d y=\frac{1}{\left|B_{\lambda_{2}}\right|} \int_{B_{\lambda_{2}}(x)} u(y) d y-\frac{1}{\left|B_{\lambda_{1}}\right|} \int_{B_{\lambda_{1}}(x)} u(y) d y
$$

and so

$$
\frac{1}{\left|B_{\lambda_{1}}\right|} \int_{B_{\lambda_{1}}(x)} u(y) d y \geq \frac{1}{\left|B_{\lambda_{2}}\right|} \int_{B_{\lambda_{2}}(x)} u(y) d y-\|\left.\Delta u\right|_{L^{p}\left(B_{R+1}\right)}|| \Gamma_{\lambda_{1}}-\Gamma_{\lambda_{2}}||_{L^{q}\left(B_{\lambda_{2}}\right)} .
$$

Using the monotonicity of $\Gamma_{\lambda}$ with respect to $\lambda$, we have

$$
\left\|\Gamma_{\lambda_{1}}-\Gamma_{\lambda_{2}}\right\|_{L^{q}\left(B_{\lambda_{2}}\right)} \leq\left\|\Gamma_{\lambda_{1}}\right\|_{L^{q}\left(B_{\lambda_{2}}\right)} \leq\|V\|_{L^{q}\left(B_{\lambda_{2}}\right)}
$$

where a simple computation yields

$$
\|V\|_{L^{q\left(B_{\lambda_{2}}\right)}}^{q}=C \int_{B_{\lambda_{2}}} \frac{1}{|x|^{(N-2) q}} d x=C \int_{0}^{\lambda_{2}} r^{N-1-(N-2) q} d r=C \lambda_{2}^{N-(N-2) q}
$$


when $N \geq 3$, and

$$
\|V\|_{L^{q}\left(B_{\lambda_{2}}\right)}^{q}=\left.C \int_{B_{\lambda_{2}}}|\log | x\right|^{q} d x=C \int_{0}^{\lambda_{2}}|\log r|^{q} r d r \leq\left|\log \lambda_{2}\right|^{q} \lambda_{2}^{2}
$$

when $N=2$. We deduce that

$$
\frac{1}{\left|B_{\lambda_{1}}\right|} \int_{B_{\lambda_{1}}(x)} u(y) d y \geq \frac{1}{\left|B_{\lambda_{2}}\right|} \int_{B_{\lambda_{2}}(x)} u(y) d y-C \lambda_{2}^{\alpha}
$$

with $\alpha=2-\frac{N}{p}$ when $N \geq 3$ (and the corresponding inequality when $N=2$ ). Finally, taking $\lambda_{1} \rightarrow 0$ using the continuity of $u$ (given by Sobolev's embedding theorems), we obtain the desired result.

Remark A.1. If we can assure that $u(x) \geq \lim _{r \rightarrow 0^{+}} \frac{1}{\left|B_{r}\right|} \int_{B_{r}(x)} u(y) d y$ for every $x$, then we can replace the hypothesis of $\Delta u \in L_{l o c}^{p}\left(\mathbb{R}^{N}\right)$, by $(\Delta u)_{+} \in L_{l o c}^{p}\left(\mathbb{R}^{N}\right)$.

\section{B. Fractional Mean Value Formula}

We now recall the derivation of the mean-value formula in the fractional case (see [51]):

Proof of Lemma 6.2. Take $0<\lambda_{1}<\lambda_{2}<d(x, \partial \Omega)$, then the function $\Gamma_{\lambda_{1}}-\Gamma_{\lambda_{2}}$ is a $C^{1,1}$ function, which is non-negative and compactly supported in $\Omega$. As in the proof of the usual mean-value formula, we now consider the integral

$$
\int_{\Omega}(-\Delta)^{s} u(x+y)\left(\Gamma_{\lambda_{1}}(y)-\Gamma_{\lambda_{2}}(y)\right) d y
$$

An integration by parts yields

$$
\int_{\Omega}(-\Delta)^{s} u(x+y)\left(\Gamma_{\lambda_{1}}(y)-\Gamma_{\lambda_{2}}(y)\right) d y=u * \gamma_{\lambda_{1}}(x)-u * \gamma_{\lambda_{2}}(x) .
$$

If $(-\Delta)^{s} u \geq 0$ in $\Omega$, then the integrand in the left had side is always non-negative and we immediately deduce

$$
u * \gamma_{\lambda_{1}}(x) \geq u * \gamma_{\lambda_{2}}(x)
$$

Taking the limit $\lambda_{1} \rightarrow 0$ (using the upper semi-continuity of $u$ ), we obtain

$$
u(x) \geq u * \gamma_{\lambda_{2}}(x)
$$

which is the first part of the lemma.

If now $(-\Delta)^{s} u \in L_{l o c}^{p}\left(\mathbb{R}^{N}\right)$, then we use Hölder inequality to get

$\left|\int_{\mathbb{R}^{N}}(-\Delta)^{s} u(x+y)\left(\Gamma_{\lambda_{1}}(y)-\Gamma_{\lambda_{2}}(y)\right) d y\right| \leq\left\|(-\Delta)^{s} u\right\|_{L^{p}\left(B_{1}(x)\right)}|| \Gamma_{\lambda_{1}}-\Gamma_{\lambda_{2}} \|_{L^{q}\left(B_{\lambda_{2}}(0)\right)}$.

We deduce from the first part of the Lemma that

$$
u(x) \geq u * \gamma_{\lambda_{1}}(x) \geq u * \gamma_{\lambda_{2}}(x)-\left\|(-\Delta)^{s} u\right\|_{L^{p}\left(B_{1}(x)\right)}|| \Gamma_{\lambda_{1}}-\Gamma_{\lambda_{2}} \|_{L^{q}\left(B_{\lambda_{2}}\right)} .
$$


Using the monotonicity of $\Gamma_{\lambda}$ with respect to $\lambda$, we see that $0 \leq \Gamma_{\lambda_{1}}-\Gamma_{\lambda_{2}} \leq \Gamma_{\lambda_{1}}$ and so

$$
\left\|\Gamma_{\lambda_{1}}-\Gamma_{\lambda_{2}}\right\|_{L^{q}\left(B_{\lambda_{2}}\right)} \leq\left\|\Gamma_{\lambda_{1}}\right\|_{L^{q}\left(B_{\lambda_{2}}\right)} \leq\left\|V_{S}\right\|_{L^{q}\left(B_{\lambda_{2}}\right)} .
$$

Finally, a simple computation yields

$$
\left\|V_{s}\right\|_{L^{q\left(B_{\lambda_{2}}\right)}}^{q}=c_{N, s}^{q} \int_{B_{\lambda_{2}}} \frac{1}{|x|^{(N-2 s) q}}=C \int_{0}^{\lambda_{2}} r^{N-1-(N-2 s) q} d r=C \lambda_{2}^{N-(N-2) q}
$$

for some constant $C$ depending only on $N, s$ and $q$. We conclude that

$$
u(x) \geq u * \gamma_{\lambda_{2}}(x)-C\left\|(-\Delta)^{s} u\right\|_{L^{p}\left(B_{1}(x)\right)} \lambda_{2}^{\alpha},
$$

which is the desired result.

\section{References}

1. Albi, G., Balagué, D., Carrillo, J.A., von Brecht, J.: Stability analysis of flock and mill rings for 2nd order models in swarming. SIAM J. Appl. Math. 74, 794-818 (2014)

2. Balagué, D., Carrillo, J.A., Laurent, T., Raoul, G.: Nonlocal interactions by repulsive-attractive potentials: Radial ins/stability. Physica D 260, 5-25 (2013)

3. Balagué, D., Carrillo, J.A., Laurent, T., Raoul, G.: Dimensionality of local minimizers of the interaction energy. Arch. Rat. Mech. Anal. 209(3), 1055-1088 (2013)

4. Balagué, D., Carrillo, J.A., Yao, Y.: Confinement for repulsive-attractive kernels. DCDS-B 19, 12271248 (2014)

5. Bernoff, A.J., Topaz, C.M.: A primer of swarm equilibria. SIAM J. Appl. Dyn. Syst. 10(1), 212-250 (2011)

6. Bertozzi, A., Carrillo, J.A., Laurent, T.: Blowup in multidimensional aggregation equations with mildly singular interaction kernels. Nonlinearity 22, 683-710 (2009)

7. Bertozzi, A.L., Laurent, T., Léger, F.: Aggregation and spreading via the newtonian potential: the dynamics of patch solutions. Math. Models Methods Appl. Sci. 22(supp01), 1140005 (2012)

8. Blank, I.: Sharp results for the regularity and stability of the free boundary in the obstacle problem. Indiana Univ. Math. J. 50, 1077-1112 (2001)

9. Brézis, H., Kinderlehrer, D.: The smoothness of solutions to nonlinear variational inequalities. Indiana Univ. Math. J. 23, 831-844 (1974)

10. Caffarelli, L.A.: The obstacle problem revisited. J. Fourier Anal. Appl. 44, 383-402 (1998)

11. Caffarelli, L.A.: A remark on the Hausdorff measure of a free boundary, and the convergence of coincidence sets. Boll. Un. Mat. Ital. A 18.1, 109-113 (1981)

12. Caffarelli, L.A., Dolbeault, J., Markowich, P.A., Schmeiser, C.: On Maxwellian equilibria of insulated semiconductors. Interfaces Free Bound. 2, 331-339 (2000)

13. Caffarelli, L.A., Friedman, A.: A singular perturbation problem for semiconductors. Boll. Un. Mat. Ital. B 7.1, 409-421 (1987)

14. Caffarelli, L.A., Salsa, S., Silvestre, L.: Regularity estimates for the solution and the free boundary of the obstacle problem for the fractional Laplacian. Inventiones Mathematicae 171, 425-461 (2008)

15. Caffarelli, L.A., Vázquez, J.L.: Nonlinear porous medium flow with fractional potential pressure. Arch. Ration. Mech. Anal. 202, 537-565 (2011)

16. Caffarelli, L.A., Vázquez, J.L.: Asymptotic behaviour of a porous medium equation with fractional diffusion. Discrete Contin. Dyn. Syst. 29, 1393-1404 (2011)

17. Cañizo, J.A., Carrillo, J.A., Patacchini, F.S.: Existence of global minimisers for the interaction energy. Arch. Rat. Mech. Anal. 217, 1197-1217 (2015)

18. Carrillo, J.A., Chertock, A., Huang, Y.: A finite-volume method for nonlinear nonlocal equations with a gradient flow structure. Commun. Comput. Phys. 17, 233-258 (2015)

19. Carrillo, J.A., Chipot, M., Huang, Y.: On global minimizers of repulsive-attractive power-law interaction energies. Philos. Trans. R. Soc. A 372, 20130399 (2014)

20. Carrillo, J.A., Di Francesco, M., Figalli, A., Laurent, T., Slepčev, D.: Global-in-time weak measure solutions and finite-time aggregation for nonlocal interaction equations. Duke Math. J. 156, 229-271 (2011)

21. Carrillo, J.A., Di Francesco, M., Figalli, A., Laurent, T., Slepčev, D.: Confinement in nonlocal interaction equations. Nonlinear Anal. 75(2), 550-558 (2012) 
22. Carrillo, J.A., Ferreira, L.C.F., Precioso, J.C.: A mass-transportation approach to a one dimensional fluid mechanics model with nonlocal velocity. Adv. Math. 231(1), 306-327 (2012)

23. Carrillo, J.A., Huang, Y., Martin, S.: Nonlinear stability of flock solutions in second-order swarming models. Nonlinear Anal. Real World Appl. 17, 332-343 (2014)

24. Carrillo, J.A., McCann, R.J., Villani, C.: Kinetic equilibration rates for granular media and related equations: entropy dissipation and mass transportation estimates. Rev. Mat. Iberoamericana 19(3), 9711018 (2003)

25. Carrillo, J.A., McCann, R.J., Villani, C.: Contractions in the 2-wasserstein length space and thermalization of granular media. Arch. Rat. Mech. Anal. 179, 217-263 (2006)

26. Chafaï, D., Gozlan, N., Zitt, P.-A.: First order global asymptotics for confined particles with singular pair repulsion. Ann. Appl. Probab. 24, 2371-2413 (2014)

27. Choksi, R., Fetecau, R., Topaloglu, I.: On minimizers of interaction functionals with competing attractive and repulsive potentials. Ann. Inst. H. Poincaré Anal. Non Linéaire 32, 1283-1305 (2015)

28. D’Orsogna, M.R., Chuang, Y., Bertozzi, A., Chayes, L.: Self-propelled particles with soft-core interactions: patterns, stability and collapse. Phys. Rev. Lett. 96, (104302) (2006)

29. Doye, J.P.K., Wales, D.J., Berry, R.S.: The effect of the range of the potential on the structures of clusters. J. Chem. Phys. 103, 4234-4249 (1995)

30. Fellner, K., Raoul, G.: Stable stationary states of non-local interaction equations. Math. Models Methods Appl. Sci. 20(12), 2267-2291 (2010)

31. Fellner, K., Raoul, G.: Stability of stationary states of non-local equations with singular interaction potentials. Math. Comput. Model. 53(7-8), 1436-1450 (2011)

32. Fetecau, R.C., Huang, Y., Kolokolnikov, T.: Swarm dynamics and equilibria for a nonlocal aggregation model. Nonlinearity 24(10), 2681-2716 (2011)

33. Fetecau, R.C., Huang, Y.: Equilibria of biological aggregations with nonlocal repulsive-attractive interactions. Physica D 260, 49-64 (2013)

34. Frostman, O.: Potentiel d'Equilibre et Capacité des Ensembles. Ph.D. thesis, Faculté des Sciences de Lund (1935)

35. Givens, C.R., Shortt, R.M.: A class of Wasserstein metrics for probability distributions. Michigan Math. J. 31(2), 231-240 (1984)

36. Gustafsson, B.: A simple proof of the regularity theorem for the variational inequality of the obstacle problem. Nonlinear Anal. 12(10), 1487-1490 (1986)

37. Hagan, M.F., Chandler, D.: Dynamic pathways for viral capsid assembly. Biophys. J. 91, $42-54$ (2006)

38. Kolokolnikov, T., Carrillo, J.A., Bertozzi, A., Fetecau, R., Lewis, M.: Emergent behaviour in multi-particle systems with non-local interactions. Physica D: Nonlinear Phenomena 260, 1-4 (2013)

39. Kinderlehrer, D., Stampacchia, G.: An Introduction to Variational Inequalities and Their Applications, vol. 88 of Pure and Applied Mathematics. Academic Press, New York-London (1980)

40. Li, H., Toscani, G.: Long-time asymptotics of kinetic models of granular flows. Arch. Rat. Mech. Anal. 172(3), 407-428 (2004)

41. López-García, A.: Greedy energy points with external fields. Recent trends in orthogonal polynomials and approximation theory, Contemp. Math., vol. 507, pp. 189-207. Amer. Math. Soc., Providence, RI (2010)

42. Mattila, P.: Geometry of sets and measures in Euclidean spaces, vol. 44 of Cambridge Studies in Advanced Mathematics. Cambridge University Press, Cambridge (1995). Fractals and rectifiability.

43. Mogilner, A., Edelstein-Keshet, L.: A non-local model for a swarm. J. Math. Bio. 38, 534-570 (1999)

44. Mogilner, A., Edelstein-Keshet, L., Bent, L., Spiros, A.: Mutual interactions, potentials, and individual distance in a social aggregation. J. Math. Biol. 47(4), 353-389 (2003)

45. Otto, F.: The geometry of dissipative evolution equations: the porous medium equation. Commun. Partial Differ. Equ. 26, 101-174 (2001)

46. Petz, D., Hiai, F.: Logarithmic energy as an entropy functional. Advances in differential equations and mathematical physics (Atlanta, GA, 1997). Contemp. Math., vol. 217, pp. 205-221. Amer. Math. Soc., Providence, RI (1998)

47. Raoul, G.: Non-local interaction equations: Stationary states and stability analysis. Differential Integral Equations 25(5-6), 417-440 (2012)

48. Rechtsman, M.C., Stillinger, F.H., Torquato, S.: Optimized interactions for targeted self-assembly: application to a honeycomb lattice. Phys. Rev. Lett. 95, 22 (2005)

49. Ruelle, D.: Statistical Mechanics: Rigorous Results. W. A. Benjamin Inc, New York-Amsterdam (1969)

50. Serfaty, S., Vázquez, J.L.: Hydrodynamic limit of nonlinear diffusions with fractional Laplacian operators. Calc. Var. PDE (2013)

51. Silvestre, L.: Regularity of the obstacle problem for a fractional power of the Laplace operator. Commun. Pure Appl. Math. 60, 67-112 (2007)

52. Simione, R., Slepčev, D., Topaloglu, I.: Existence of minimizers of nonlocal interaction energies. J. Stat. Phys. 159, 972-986 (2015) 
53. Stein, E.: Singular Integrals and Differentiability Properties of Functions. Princeton University Press, Princeton (1970)

54. Theil, F.: A proof of crystallization in two dimensions. Commun. Math. Phys. 262, 209-236 (2006)

55. Villani, C.: Topics in Optimal Transportation, vol. 58 of Graduate Studies in Mathematics. American Mathematical Society, Providence, RI (2003)

56. von Brecht, J., Uminsky, D.: On soccer balls and linearized inverse statistical mechanics. J. Nonlinear Sci. 22(6), 935-959 (2012)

57. Wales, D.J.: Energy landscapes of clusters bound by short-ranged potentials. Chem. Eur. J. Chem. Phys. 11, 2491-2494 (2010)

Communicated by C. Mouhot 